\title{
8. DIAGENESIS AND HYDROLOGY AT THE NEW HEBRIDES FOREARC AND INTRA-ARC AOBA BASIN ${ }^{1}$
}

\author{
Jonathan B. Martin ${ }^{2}$
}

\begin{abstract}
Depending on the temperature and the extent of diagenetic alteration of fluid chemistry, fluid flow at convergent margins may transfer important quantities of heat and mass between the crust and seawater, thereby influencing global mass, isotopic and heat budgets. In the North Aoba Basin, an intra-arc basin located at the New Hebrides Island Arc, alteration of volcanic ash to clay minerals and zeolites forms a $\mathrm{CaCl}_{2}$ brine, perhaps in less than 1 to 3 m.y. The brine results from an exchange of $\mathrm{Ca}$ for $\mathrm{Na}, \mathrm{K}$, and $\mathrm{Mg}$, and an increase in $\mathrm{Cl}$ concentrations to a maximum of $1241 \mathrm{mM}$. The $\mathrm{Cl}$ increase is partly due to the transfer of $\mathrm{H}_{2} \mathrm{O}$ from the pore fluid into authigenic minerals, but water mass balances, $\delta^{18} \mathrm{O}-\mathrm{Cl}$ correlations, and $\mathrm{Br} / \mathrm{Cl}$ ratios suggest that there is a source of $\mathrm{Cl}$ in the sediments. Concentration profiles indicate that $\mathrm{Li}$ is transferred from the fluid to solid phase at depths $<300$ meters below seafloor (mbsf), but at greater depths it is transferred from the solid to fluid phase, at temperatures possibly as low as $25^{\circ} \mathrm{C}$.

In the accretionary wedge extensive fluid flow appears to be confined to highly faulted regions. Although $\mathrm{Cl}$ concentrations less than seawater value are common at convergent margins, the New Hebrides margin contains little low- $\mathrm{Cl}$ fluid. $\mathrm{Br} / \mathrm{Cl}$ ratios suggest the low- $\mathrm{Cl}$ fluid is from dilution, and $\delta^{18} \mathrm{O}$ values indicate the water may be derived from mineral dehydration and mixing with meteoric water. The New Hebrides margin exhibits few surface manifestations of venting (e.g., sulfide-oxidizing benthic biological communities, carbonate crusts, mud volcanoes) and thus fluid fluxes may be smaller than at many other margins.
\end{abstract}

\section{INTRODUCTION}

At convergent margins, the flow of fluids between Layers I and II of the oceanic crust and seawater could carry important fluxes of heat and mass, thereby influencing their global distributions (Han and Suess, 1989; Kastner et al., 1991; Martin et al., 1991). The origins of the fluids and the magnitudes of their fluxes are not precisely known. Some fluid flow is caused simply by porosity reduction during tectonic compaction of the accreted sediment (von Huene and Lee, 1983; Bray and Karig, 1985; Fowler et al., 1985; Carson et al., 1990); this flow is estimated to flux to the oceans at the rate of $\sim 1 \mathrm{~km}^{3}$ water/yr (COSOD II, 1987). At certain convergent margins, however, flow volumes are greater than those that can be sustained by porosity reduction alone, implying that some fluids originate from regions external to the margin (Le Pichon et al., 1990, 1991; Kastner et al., 1991). The magnitudes of heat and mass fluxes depend directly on the volumes, temperatures and compositions of the advecting fluids. Because temperatures generally increase with burial depth, vertical fluid advection would transport the greatest quantities of heat. The depth distributions of fluid compositions are more complex than the heat distributions and depend on the origins of the fluids, on their hydrology, and on several important fluid-solid reactions. For any particular margin, the dominating fluid-solid reactions are controlled by the types of basement rocks and sediments, while the reaction rates are controlled by the temperatures. Although a variety of sediment types is found at convergent margins, every convergent margin is a site of active volcanism, and ash is an important component of all margin sediments. The ash is diluted to various degrees by other components, commonly biogenic and terrigenous detritus. Because basaltic ash is highly unstable in the marine environment, its alteration is one of the important fluid-solid reactions altering pore-fluid chemistry, even when greatly diluted by other phases.

\footnotetext{
'Greene, H.G., Collot, J.-Y., Stokking, L.B., et al., 1994. Proc. ODP, Sci. Results, 134: College Station, TX (Ocean Drilling Program).

${ }^{2}$ Scripps Institution of Oceanography, University of California-San Diego, La Jolla, CA 92093-0208, U.S.A.
}

The tectonic setting and sedimentology of the New Hebrides convergent margin (Fig. 1; Collot et al., 1985; Greene and Wong, 1988; Collot and Fisher, 1991; Fisher et al., 1991; Collot, Greene, Stokking, et al., 1992) provide an ideal location to study fluid flow in forearc sediments and alteration of pore fluid chemistry caused by volcanic ash diagenesis. Sediments at the margin are composed mostly of volcanic ash and breccias and biogenic carbonate (Fig. 2). Collision of the d'Entrecasteaux Zone (DEZ; Daniel and Katz, 1981; Collot et al., 1985; Marthelot et al., 1985) contributes to the accretion of material from the subducting Australian Plate onto the overriding Pacific Plate and creates rapid porosity reduction within the accretionary wedge (Leonard and Ask, this volume). The collision also uplifts Espiritu Santo and Malakula islands (Taylor, 1992) thereby forming the intra-arc Aoba Basin (Fig. 1). This basin traps sediments that are rapidly deposited at rates of 100 to $300 \mathrm{~m} / \mathrm{m}$.y. (Collot, Greene, Stokking, et al., 1992). Because there is less deviatoric stress in the basin than in the accretionary wedge and only a small hydrologic head from the surrounding islands, there should be little or no lateral fluid flow through the basin sediments. Pore-fluid concentrations that deviate from seawater values therefore would result solely from fluid-solid reactions and subsequent diffusion. The dominating fluid-solid reactions thus would be reflected in the pore-fluid concentration gradients and the quantities and types of authigenic minerals. Because similar sediment types are found in the North Aoba Basin and the accretionary wedge (Fig. 2), the same diagenetic reactions would be important in both regions. In the accreted sediment, deviations from the predicted pore-fluid compositional changes based on the important diagenetic reactions could indicate horizons of fluid flow and the presence of fluids with origins external to the accretionary wedge. The chemical and isotopic compositions would reflect the origins of these externally derived fluids.

Specific problems to be addressed in this paper include documenting the important diagenetic reactions and the resulting chemical and isotopic changes in non-advecting pore fluids, determining the major authigenic phases formed during these reactions, estimating a mass balance for the exchange of water between the fluid and solid phases, and relating the reactions to the temperatures based on the observed geothermal gradients. Additional important problems, specific to the accretionary wedge pore fluids, include identifying horizons of fluid 


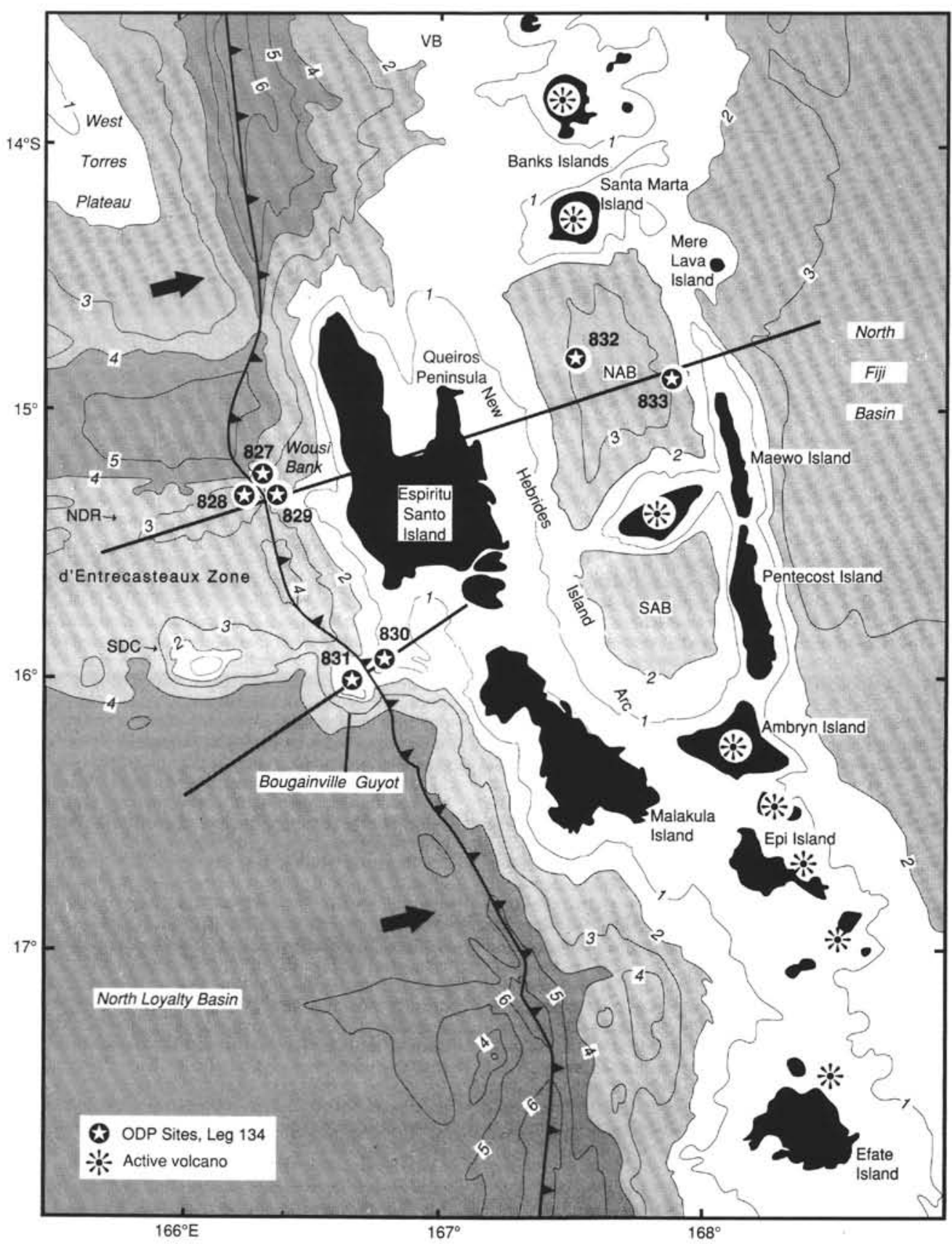

Figure 1. Map of the central New Hebrides Island Arc showing Leg 134 drill sites. Bathymetry in kilometers. NDR = North d'Entrecasteaux Ridge; SDC = South d'Entrecasteaux Chain; $\mathrm{NAB}=$ North Aoba Basin; $\mathrm{SAB}=$ South Aoba Basin. Bold line with teeth indicates approximate position of subduction zone; teeth are on upper plate. Arrows indicate direction of plate convergence. From Collot, Greene, Stokking, et al. (1992).

circulation and identifying the origins and estimating the quantity of the externally derived fluids.

\section{METHODS AND SAMPLE HANDLING}

The pore fluids were separated from the sediment by squeezing 5 to $35-\mathrm{cm}$ lengths of the whole round core in a mechanical vice at pressures up to $2 \times 10^{8} \mathrm{~Pa}$. Based on visual inspection, portions of each solid sample that appeared to be contaminated with drilling fluid (surface seawater) were physically separated from the pristine sediment. Some samples were collected and squeezed but were too con- taminated for use in this study; these are listed in Collot, Greene, Stokking, et al. (1992). After separation, the fluid was filtered through a $0.45 \mu \mathrm{m}$ filter prior to storage or analysis. A portion of the separated fluid was used for shipboard measurements of $\mathrm{Cl}, \mathrm{Na}, \mathrm{Ca}, \mathrm{Mg}, \mathrm{NH}_{4}$, $\mathrm{PO}_{4}, \mathrm{Si}, \mathrm{SO}_{4}, \mathrm{pH}$, alkalinity and salinity concentrations, and the remaining fluid was archived for future shore-based analytical work. The sampling procedure is described by Collot, Greene, Stokking, et al. (1992). The techniques used for shipboard analyses are standard shipboard procedures described by Gieskes et al. (1992).

The shore-based geochemical analyses include measurements of the pore-fluid concentrations of $\mathrm{Li}, \mathrm{Sr}, \mathrm{Br}, \mathrm{I}$, and $\mathrm{B}$ from all seven 
Bougainville Guyot and d'Entrecasteaux Ridge

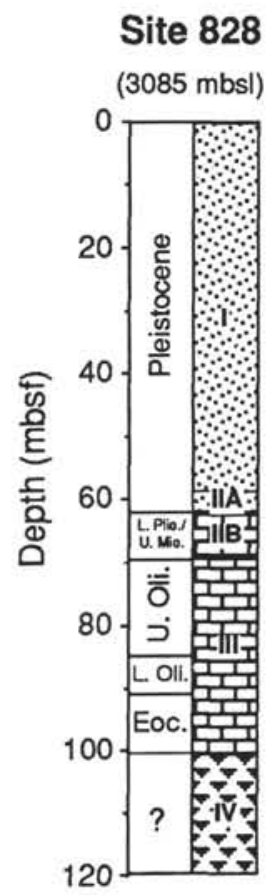

\section{Aoba Basin}

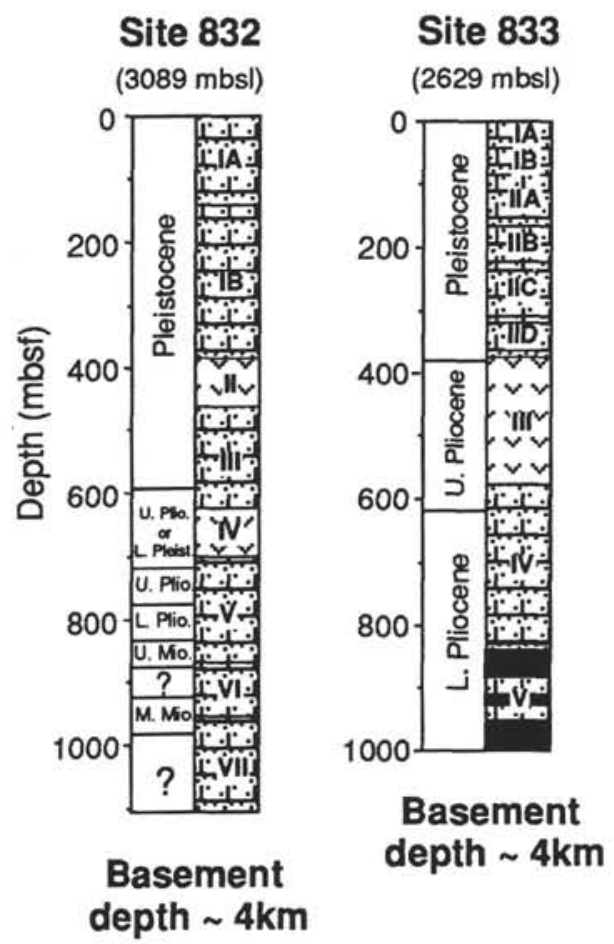

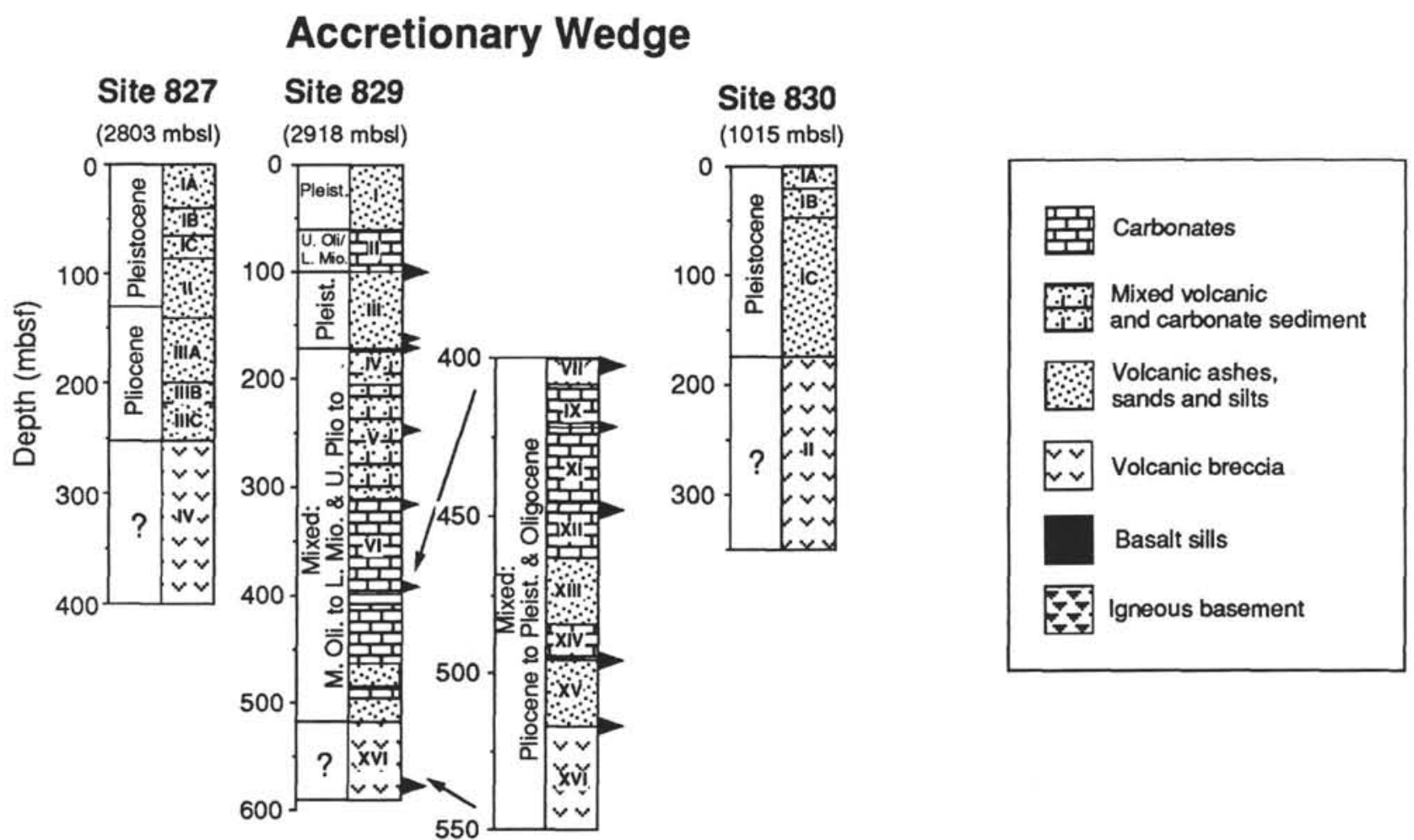

Figure 2. Schematic diagram of general lithologies and sedimentary units at the New Hebrides Island Arc. Water depth for each site, shown as meters below sea level (mbsl), is the average of the water depths of all holes at each site. At Site 829 large arrows indicate major thrust faults; small arrows indicate minor thrust faults. Units VIII and X are 0.4 and $5.7 \mathrm{~m}$ thick, respectively, and are not shown. Data taken from Collot, Greene, Stokking, et al. (1992). 
sites. The ${ }^{87} \mathrm{Sr} /{ }^{86} \mathrm{Sr}$ ratios of dissolved $\mathrm{Sr}$ and $\delta^{18} \mathrm{O}$ and $\delta \mathrm{D}$ values of the water were measured for Sites 827 and 829 in the accretionary wedge and for Site 833 in the Aoba Basin. The Li and Sr concentrations were measured by flame atomic emission and absorption spectroscopy respectively, $\mathrm{Br}$ concentrations by an iodiometric titration, and the I and B concentrations spectrophotometrically (Gieskes et al., 1992). Based on repeated measurements of either IAPSO or La Jolla Pier Water used as internal standards, the precision of all solute measurements is within $\pm 4 \%(2 \sigma)$. The ${ }^{87} \mathrm{Sr} /{ }^{86} \mathrm{Sr}$ ratios were measured at Scripps Institution of Oceanography with standard values of 0.710265 for NBS 987 and 0.709175 for North Atlantic and Central Pacific seawater. Repeated measurements of these standards yield a standard error of $\pm 22 \times 10^{-6}(2 \sigma)$. The $\delta \mathrm{D}$ and $\delta^{18} \mathrm{O}$ values were measured at Global Geochemical Corporation and are reported relative to the SMOW standard with errors of $\pm 1.5 \%(2 \sigma)$ for the $\delta D$ values and $\pm 0.1 \%$ o $(2 \sigma)$ for the $\delta^{18} O$ values.

The mineralogical compositions of selected bulk sediments from Sites 829,832 , and 833 were determined by X-ray diffraction techniques $\left(\mathrm{Cu} \mathrm{K}_{\alpha}\right.$ radiation) using aliquots of the sediments from which the pore fluids had been extracted. No treatments were applied to the sediments prior to the analyses. The relative mineralogical abundances in each sample were qualitatively estimated based on the major peak heights of each mineral.

\section{RESULTS}

The concentrations of all the measured solutes from each site are given in Table 1, which reports the concentrations at the North d'Entrecasteaux Ridge (NDR) and Bougainville Guyot Sites 828 and 831 , the concentrations at the North Aoba Basin Sites 832 and 833, and the concentrations at the accretionary wedge Sites 827,829 , and 830 . Figure 3 shows the depth profile at the NDR Site 828 , but because only two samples were collected from the Bougainville Guyot (Table 1 , Sites 828,831 ), these data are not plotted vs. depth. Table 2 reports the $\delta \mathrm{D}$ and $\delta^{18} \mathrm{O}$ values of water and Table 3 reports the ${ }^{87} \mathrm{Sr} /{ }^{86} \mathrm{Sr}$ ratios of dissolved $\mathrm{Sr}$ at Sites 827,829 , and 833. Based on the results of $\mathrm{X}$-ray diffraction measurements, bulk mineralogy of selected samples are reported in Table 4.

\section{DISCUSSION}

\section{North d'Entrecasteaux Ridge and Bougainville Guyot (Sites 828 and 831)}

Site 828 , drilled on the crest of the NDR (Fig. 1), is a reference site designed to provide information about the sediments approaching the subduction zone. The objectives of this site were to document transfer of material from the subducted to the overriding plate and to observe whether fluids flow from the accretionary wedge into sediments seaward of the deformation front. Similarity between sediments found at Site 828 and the accretionary wedge Site 829 (Fig. 2) suggests that sediment has been transferred from the ridge into portions of the accretionary wedge (Collot, Greene, Stokking, et al., 1992; Reid et al., this volume). At Site 828 , solute concentrations vary only slightly from seawater values (Fig. 3) and these compositional changes can best be explained as a result of various diagenetic reactions. At shallow burial depths ( $<18.4$ meters below seafloor [mbsf]), maxima in $\mathrm{NH}_{4}$, alkalinity, and phosphate, and a slight decrease (to $26.3 \mathrm{mM}$ ) in the $\mathrm{SO}_{4}$ concentration demonstrate minor organic carbon diagenesis. At greater burial depths (below 50 mbsf) decreasing $\mathrm{Mg}$ and increasing $\mathrm{Ca}$ concentrations indicate reaction with the basement rocks or ash, typical of the alteration from basalt to clay minerals (McDuff and Gieskes, 1976; Lawrence and Gieskes, 1981; Gieskes, 1981). These reactions could also be responsible for the decreasing $\mathrm{K}$ and $\mathrm{Na}$ concentrations. The $\mathrm{Sr}$ concentration increases from the sediment/water interface to the basement. This increase is sharpest in the deepest two samples reflecting the increasing sedimentary carbonate content (Fig. 2). The $\mathrm{Li}$ and $\mathrm{B}$ concentrations exhibit shallow minima that could reflect uptake reactions during ash diagenesis. None of these changes in the pore-fluid chemistry could be attributed to flow of fluid from the accretionary wedge. No section of tectonized sediment was observed which would indicate the location of the proto-decollement and the likely flow horizon for accretionary wedge fluids. In contrast to the New Hebrides margin, at the Barbados margin chemical characteristics of fluids recovered from the proto-decollement zone and from sand layers below the proto-decollement seaward of the deformation front suggest that there is active flow from the accretionary wedge (Moore, Mascle, et al., 1987; Blanc et al., 1988; 1991; Gieskes et al., 1990; Vrolijk et al., 1990, 1991).

The Bougainville Guyot site (831) was plagued by limited core recovery and thus only two pore-fluid samples were collected from the 727-m thick carbonate cap (Fig. 2). These samples are characterized by solute concentrations that are close to seawater values, with the exception of elevated $\mathrm{Sr}$ concentrations of $136.7 \mu \mathrm{M}$ at $3 \mathrm{mbsf}$ and $212.8 \mu \mathrm{M}$ at $135 \mathrm{mbsf}$ (Table 1). These concentrations probably reflect recrystallization of carbonate, also observed by Quinn and Taylor (this volume). Even the shallowest fluid sample is characterized by a Sr concentration $56 \%$ greater than seawater value, suggesting there is a diffusive $\mathrm{Sr}$ flux upward through the guyot.

\section{North Aoba Basin (Sites 832 and 833)}

\section{Diagenetic and Temperature Controls of Mineral Authigenesis and Solute Concentrations}

In the North Aoba Basin Sites 832 and 833, all pore fluid solutes show large deviations from seawater concentrations (Figs. 4 and 5). The sediment contains an average of $<0.5 \mathrm{wt} \%$ organic carbon (Collot, Greene, Stokking, et al., 1992), and at burial depths above 100 mbsf where the sediment was deposited at rates $>300 \mathrm{~m} / \mathrm{m}$.y., maxima in the alkalinity, $\mathrm{PO}_{4}, \mathrm{NH}_{4}$, and $\mathrm{I}$ concentrations and a corresponding $\mathrm{SO}_{4}$ minimum reflect organic carbon diagenesis. In only a few samples at Site 833 is $\mathrm{SO}_{4}$ reduction complete, and at Site 832 the $\mathrm{SO}_{4}$ minimum reaches values of only $0.6 \mathrm{mM}$ (Table 1 ). Below these minima, the concentrations increase to maxima of around $23.8 \mathrm{mM}$ (Site 832) and $14.6 \mathrm{mM}$ (Site 833), approaching but never reaching seawater concentration. At the depths of the $\mathrm{SO}_{4}$ maxima, the sedimentary organic-carbon contents are often $<0.1 \mathrm{wt} \%$ (Collot, Greene, Stokking, et al., 1992) and the $\mathrm{PO}_{4}$ and $\mathrm{NH}_{4}$ concentrations are low-less than $1 \mu \mathrm{M}$ and $350 \mu \mathrm{M}$, respectively (Figs. 4 and 5). These low organic carbon, $\mathrm{PO}_{4}$, and $\mathrm{NH}_{4}$ concentrations imply that there has been little organic carbon available for diagenesis, hence minor $\mathrm{SO}_{4}$ reduction. The $\mathrm{SO}_{4}$ maxima thus could be caused by trapping of seawater sulfate in organic carbon-poor sediment. Sediment between the $\mathrm{SO}_{4}$ maxima and minima was deposited in less than $1 \mathrm{~m} . \mathrm{y}$. (Collot, Greene, Stokking, et al., 1992), and thus little $\mathrm{SO}_{4}$ has diffused into the overlying, more organic carbon-rich sediment.

Sediments at those burial depths with $\mathrm{SO}_{4}$ maxima correspond to lithostratigraphic units composed principally of volcanic breccias: Units II and IV at Site 832 and Unit III at Site 833 (Fig. 2), which diluted to even lower values the small quantities of detrital organic matter found in the other units. Similar to the diagenesis attributed to basement reactions on the NDR, diagenesis of the volcanic breccia strongly alters the alkali and alkaline earth-element concentrations. Because the sediment thickness in the North Aoba Basin reaches a maximum of $4 \mathrm{~km}$ (Pontoise et al., this volume), this diagenesis must result from reactions within the sediments. The amount of volcanic material and the sedimentation rates are greater in the North Aoba Basin than on the NDR, and thus the solute concentrations are more altered in the North Aoba Basin. Within the volcanic breccia units the pore fluid contains no measurable $\mathrm{Mg}$, the $\mathrm{Na}$ and $\mathrm{K}$ concentrations decrease to minimum values (Table 1; Figs. 4 and 5), and Ca concentrations increase to maxima of $216 \mathrm{mM}$ and $549 \mathrm{mM}$ at Sites 832 and 833 , respectively. Above the breccia units, the $\mathrm{Na}$ and $\mathrm{K}$ concentrations are greater than seawater values by up to $16 \%$ and $52 \%$, possibly representing leaching of these elements from the ash at low tempera- 

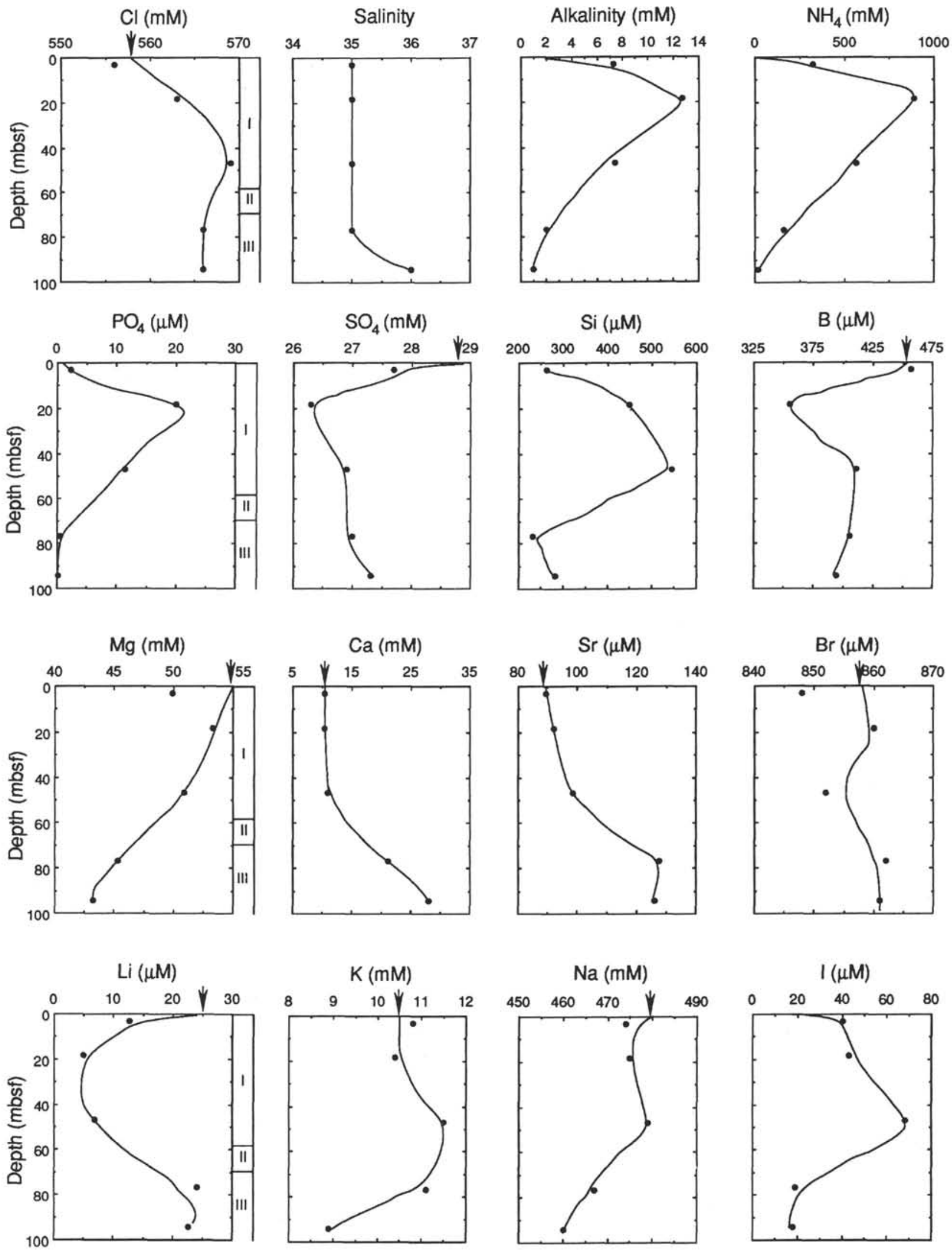

Figure 3. Depth profiles of pore fluid species and unit thicknesses for North d'Entrecasteaux Ridge Site 828. Arrows indicate seawater composition. 
Table 1. Pore fluid solute concentrations for Sites 827, 828, 829, 830, 831, 832, 833 .

\begin{tabular}{|c|c|c|c|c|c|c|c|c|c|c|c|c|c|c|c|c|c|c|}
\hline $\begin{array}{l}\text { Core, section, } \\
\text { interval }(\mathrm{cm})\end{array}$ & $\begin{array}{l}\text { Depth } \\
\text { (mbsf) }\end{array}$ & $\underset{(\mathrm{mM})}{\mathrm{Cl}}$ & Salinity & $\begin{array}{l}\text { Alkalinity } \\
(\mathrm{mM})\end{array}$ & $\mathrm{pH}$ & $\begin{array}{l}\mathrm{NH}_{4} \\
(\mu \mathrm{M})\end{array}$ & $\begin{array}{l}\mathrm{PO}_{4} \\
(\mu \mathrm{M})\end{array}$ & $\begin{array}{c}\mathrm{SO}_{4} \\
(\mathrm{mM})\end{array}$ & $\begin{array}{c}\mathrm{Si} \\
(\mu \mathrm{M})\end{array}$ & $\begin{array}{c}\text { B } \\
(\mu \mathrm{M})\end{array}$ & $\underset{(\mathrm{mM})}{\mathrm{Mg}}$ & $\underset{(\mathrm{mM})}{\mathrm{Ca}}$ & $\begin{array}{c}\mathrm{Sr} \\
(\mu \mathrm{M})\end{array}$ & $\begin{array}{c}\mathrm{Li} \\
(\mu \mathrm{M})\end{array}$ & $\underset{(\mathrm{mM})}{\mathrm{Na}}$ & $\underset{(\mathrm{mM})}{\mathrm{K}}$ & $\begin{array}{c}\mathrm{Br} \\
(\mu \mathrm{M})\end{array}$ & $\begin{array}{c}\mathrm{I} \\
(\mu \mathrm{M}\end{array}$ \\
\hline \multicolumn{19}{|l|}{$134-828 \mathrm{~A}-$} \\
\hline $1 \mathrm{H}-2,145-150$ & 3.0 & 556 & 35.0 & 7.3 & 7.8 & 323 & 2.3 & 27.7 & 263 & 457 & 49.9 & 10.4 & 89.5 & 12.6 & 474 & 10.8 & 848 & 40 \\
\hline $3 \mathrm{H}-3,145-150$ & 18.4 & 563 & 35.0 & 12.7 & 7.9 & 888 & 20.0 & 26.3 & 449 & 355 & 53.3 & 10.4 & 92.0 & 5.0 & 475 & 10.4 & 860 & 43 \\
\hline $6 \mathrm{H}-3,145-150$ & 46.9 & 569 & 35.0 & 7,4 & 7.6 & 567 & 11.4 & 26.9 & 545 & 411 & 50.9 & 10.9 & 98.6 & 6.8 & 479 & 11.5 & 852 & 68 \\
\hline $9 \mathrm{H}-5,145-150$ & 76.9 & 566 & 35.0 & 2.0 & 7.6 & 161 & 0.5 & 27.0 & 232 & 406 & 45.3 & 21.1 & 127.6 & 24.1 & 467 & 11.1 & 862 & 19 \\
\hline \multirow{2}{*}{$\begin{array}{l}134-828 \mathrm{~B}- \\
\quad 1 \mathrm{R}-3,135-150\end{array}$} & & & & & & & & & & & & & & & & & & \\
\hline & 94.4 & 566 & 36.0 & 1.0 & 7.5 & 18 & 0.2 & 27.3 & 282 & 394 & 43.2 & 28.0 & 125.9 & 22.6 & 460 & 8.9 & 861 & 18 \\
\hline $\begin{array}{l}134-831 \mathrm{~A}- \\
1 \mathrm{H}-2,145-150\end{array}$ & & & 5 & & & 5 & & & & & & & 1367 & & & & & \\
\hline \multirow{2}{*}{\multicolumn{19}{|c|}{ 134-831B- }} \\
\hline & & & & & & & & & & & & & & & & & & \\
\hline \multicolumn{19}{|l|}{$134-832 \mathrm{~A}-$} \\
\hline $1 \mathrm{H}-3,145-150$ & 4.5 & 551 & 34.5 & 4.9 & 7.9 & 71 & 12.0 & 26.1 & 396 & 396 & 50.6 & 9.3 & 84.6 & 24.2 & 477 & 12.1 & 841 & 20 \\
\hline $4 \mathrm{H}-3,145-150$ & 23.0 & 560 & 35.1 & 23.0 & 7.8 & 1336 & 75.0 & 4.5 & 425 & 453 & 46. & 3.0 & 48.0 & 18.7 & 486 & 11.7 & 898 & 46 \\
\hline $6 \mathrm{H}-3,145-150$ & 42.0 & 566 & 34.0 & 28.0 & 8.0 & 1680 & 99.7 & 0.6 & 437 & 452 & 47. & 1.9 & 46.5 & 15.9 & 483 & 10.8 & 879 & 60 \\
\hline $8 \mathrm{H}-2,144-150$ & 59.5 & 568 & 34,0 & 30.0 & 8.1 & 1973 & 104.5 & 0.7 & 425 & 440 & 46.7 & 2.3 & 47.2 & 14.2 & 484 & 10.9 & 872 & 67 \\
\hline $10 \mathrm{H}-3,143-150$ & 77.0 & 566 & 34.0 & 30.0 & 8.1 & 2307 & 119.8 & 0.8 & 542 & 525 & 43.8 & 2.2 & 44.1 & 14.3 & 489 & 10.3 & 859 & 72 \\
\hline $16 \mathrm{H}-3,145-150$ & 117.5 & 573 & 34.0 & 27.3 & 7.8 & 2291 & 89.3 & 0.8 & 484 & 470 & 42.3 & 2.3 & 45.7 & 16.4 & 489 & 9.6 & 879 & 74 \\
\hline $19 \mathrm{H}-1,145-150$ & 142.5 & 577 & 34.0 & 26.3 & 8.0 & 2246 & 86.4 & 1.2 & 530 & 435 & 42.6 & 2.5 & 48.0 & 17.1 & 490 & 9.7 & 880 & 70 \\
\hline $24 \mathrm{H}-2,145-150$ & 180.7 & 583 & 34.5 & 21.7 & 7.8 & 1897 & 42.5 & 2.4 & 501 & 400 & 42.7 & 3.4 & 61.3 & 26.9 & 495 & 9.2 & 897 & 65 \\
\hline \multicolumn{19}{|l|}{ 134-832B- } \\
\hline $9 \mathrm{R}-2,140-150$ & 222.9 & 596 & 34.2 & 11.0 & 8.0 & 2054 & 14.8 & 0.6 & 458 & 345 & 35.5 & 4.7 & 77.6 & 18.8 & 499 & 10.8 & 888 & 74 \\
\hline $15 R-2,58-68$ & 280.0 & 622 & 36.0 & 0.7 & 8.2 & 1599 & 1.0 & 2.9 & 316 & 218 & 14.2 & 30.6 & 243.2 & 26.3 & 501 & 15.2 & 931 & 65 \\
\hline $17 \mathrm{R}-1,10-20$ & 300.3 & 642 & 38.0 & 0.5 & 7.9 & 1159 & 0.7 & 4.8 & 389 & 169 & 15.7 & 54.5 & 479.4 & 34.8 & 484 & 12.6 & 980 & 66 \\
\hline $19 \mathrm{R}-1,140-150$ & 319.5 & 673 & 41.0 & 0.5 & 7.8 & 693 & 0.7 & 7.9 & 446 & 218 & 12.1 & 102.3 & 849.6 & 53.2 & 443 & 9.1 & 993 & 61 \\
\hline $21 \mathrm{R}-3,140-150$ & 341.8 & 700 & 45.0 & & & 203 & & 12.8 & 473 & & 0.0 & 168.3 & & & 392 & 4.4 & & \\
\hline $23 R-2,16-31$ & 358.2 & 692 & 45.0 & 0.4 & 8.1 & 168 & 0.5 & 13.8 & 511 & 255 & 0.0 & 177.3 & 511.3 & 55.7 & 363 & 3.9 & 1020 & 43 \\
\hline $28 \mathrm{R}-2,135-150$ & 407.8 & 674 & 46.3 & 0.9 & 8.9 & 46 & 0.5 & 17.0 & 121 & 291 & 0.0 & 192.4 & 434.7 & 50.7 & 344 & 2.3 & 1004 & 28 \\
\hline $30 \mathrm{R}-4,138-150$ & 428.0 & 653 & 43.2 & 0.5 & 8.5 & 142 & 0.5 & 21.5 & 389 & 238 & 0.0 & 140.8 & 1015.5 & 54.0 & 410 & 4.8 & 984 & 24 \\
\hline $32 \mathrm{R}-2,135-150$ & 445.8 & 645 & 42.2 & 0.3 & 8.3 & 261 & 0.5 & 22.0 & 477 & 258 & 2.9 & 116.7 & 1104.9 & 53.3 & 441 & 6 & 982 & 25 \\
\hline $34 \mathrm{R}-1,135-150$ & 462.9 & 645 & 42.0 & 0.3 & 8.3 & 429 & 0.5 & 22.0 & 379 & 222 & 2.5 & 97.4 & 1130.4 & 49.3 & 477 & 8.9 & 954 & 22 \\
\hline $37 \mathrm{R}-3,130-150$ & 494.7 & 637 & 41.0 & 0.3 & 8.1 & 386 & 0.5 & 22.0 & 404 & 248 & 3.3 & 90.5 & 1098.5 & 47.7 & 488 & 8.3 & 955 & 23 \\
\hline $40 \mathrm{R}-1,123-135$ & 520.7 & 655 & 44.0 & & & 323 & & 23.8 & 358 & 290 & 7.8 & 85.1 & 970.9 & 50.1 & 488 & 7.9 & 979 & 23 \\
\hline $42 R-2,0-13$ & 540.4 & 674 & 425 & 0.4 & 8.1 & 261 & 0.5 & 23.4 & 429 & 314 & 2.7 & 110.3 & 1162.4 & 56.7 & 481 & 6.4 & 1003 & 24 \\
\hline $45 \mathrm{R}-1,130-150$ & 569.0 & 698 & 46.2 & 0.3 & 6.7 & 301 & 0.5 & 21.3 & 439 & 319 & 0.9 & 148.4 & 1373.0 & 75.7 & 447 & 5.7 & 1036 & 25 \\
\hline $47 \mathrm{R}-1,130-150$ & 588.3 & 719 & 48.0 & 0.4 & 7.7 & 203 & 0.5 & 20.4 & 282 & 304 & 3.3 & 166.8 & 1277.2 & 77.8 & 415 & 5.1 & 1021 & 26 \\
\hline $49 \mathrm{R}-\mathrm{CC}, 0-20$ & 611.2 & 742 & 50.6 & 0.3 & 8.0 & 145 & 0.5 & 16.5 & 408 & 412 & 0.5 & 215.9 & 964.5 & 81.0 & 344 & 4.0 & 1040 & 25 \\
\hline $59 \mathrm{R}-3,130-150$ & 706.3 & 708 & 48.5 & 0.1 & 7.9 & 272 & 0.5 & 14.5 & 586 & 165 & 3.2 & 180.2 & 1207.0 & 71.1 & 374 & 4.3 & 1034 & 25 \\
\hline $30-150$ & 723.6 & 682 & 45.1 & 0.3 & 8.2 & 349 & & 14.6 & 270 & 135 & 3.9 & 150.6 & 1839.0 & 86.8 & 401 & 4.2 & 996 & 25 \\
\hline $63 \mathrm{R}-6,0-20$ & 747.3 & 678 & 44.2 & & & & & 14.4 & & & & & & & 432 & 4.1 & & \\
\hline $66 \mathrm{R}-3,130-150$ & 773.1 & 631 & 40.0 & & & 298 & & 19.9 & 156 & 283 & 9.2 & 98.4 & 1609.2 & 69.3 & 448 & 4.2 & 935 & 34 \\
\hline $69 \mathrm{R}-4,0-20$ & 802.3 & 680 & 44.0 & & & 352 & & 22.9 & 230 & 155 & 10.1 & 122.8 & 1373.0 & 70.4 & 462 & 3.9 & 990 & 41 \\
\hline $72 \mathrm{R}-4,127-146$ & 832.8 & 704 & 46.0 & & & 207 & & 20.8 & 150 & 216 & 0.0 & 147.0 & 875.1 & 66.9 & 450 & 3.6 & 1012 & 46 \\
\hline \multicolumn{19}{|l|}{$134-833 \mathrm{~A}$ - } \\
\hline $1 \mathrm{H}-3,145-150$ & 4.5 & 568 & 0 & 14.0 & 8.0 & 651 & 51.3 & 8 & 404 & 450 & 39 & 4.3 & 69.6 & 19.8 & 48 & 12.0 & 873 & 40 \\
\hline $3 \mathrm{H}-3,145-150$ & 23.5 & 596 & 35.0 & 19.9 & 7.8 & 1645 & 55.2 & 0.0 & 391 & 442 & 35.5 & 2.6 & 65.3 & 12.3 & 506 & 11.7 & 901 & 78 \\
\hline $6 \mathrm{H}-1,145-150$ & 40.7 & 603 & 35.0 & 16.9 & 8.1 & 1794 & 41.1 & 0.6 & 307 & 410 & 34.3 & 3.0 & 67.9 & 11.8 & 503 & 11.9 & 941 & 75 \\
\hline $8 \mathrm{H}-1,145-150$ & 55.5 & 613 & 36.0 & 14.4 & 8.0 & 1596 & 30.0 & 0.0 & 399 & 411 & 31.8 & 3.0 & 68.8 & 14.9 & 507 & 12.1 & 962 & 77 \\
\hline $10 \mathrm{H}-4,145-150$ & 68.9 & 617 & 36.0 & & & 1690 & 20.8 & 0.0 & 366 & 361 & 29 & 3.5 & 73.9 & 15.2 & 508 & 12.2 & 923 & 75 \\
\hline $13 \mathrm{H}-1,145-150$ & 79.5 & 620 & 36.0 & 8.8 & 8.1 & 1491 & 18.8 & 0.7 & 319 & 372 & 29.1 & 4.8 & 103.3 & 18.6 & 518 & 14.2 & 944 & 74 \\
\hline $16 \times-2,140-150$ & 95.6 & 636 & 36.3 & 2.7 & 8.2 & 1600 & 3.3 & 1.1 & 307 & 283 & 24.1 & 7.4 & 135.2 & 18.9 & 533 & 14.9 & 980 & 70 \\
\hline $21 X-2,140-150$ & 144.9 & 688 & 40.0 & 1.1 & 8.2 & 1466 & 1.9 & 3.3 & 292 & 253 & 25.9 & 15.6 & 219.9 & 25.7 & 512 & 12.9 & 1024 & 73 \\
\hline & 162.8 & 702 & & 1.6 & 7.8 & 1273 & 1.9 & 5.1 & 370 & 29 & 35.0 & 22.1 & 342.9 & 31.6 & 534 & 14.6 & 1042 & 80 \\
\hline $26 \mathrm{X}-1,92-102$ & 191.2 & 726 & 44.0 & 0.9 & 7.9 & 1223 & 1.9 & 7.2 & 378 & 316 & 35.5 & 43.1 & 810.3 & 53.2 & 521 & 14.2 & 1065 & 82 \\
\hline \multicolumn{19}{|l|}{ 134-833B- } \\
\hline $5 R-2,90-101$ & 118.2 & 660 & 37.8 & 1.0 & 8.0 & 1364 & 0.8 & 2.4 & 310 & 270 & 21.3 & 14.9 & 191.4 & 21.4 & 522 & 14.7 & 997 & 69 \\
\hline $16 \mathrm{R}-1,140-150$ & 223.5 & 761 & 46.3 & 0.5 & 7.8 & 855 & 0.6 & 10.3 & 464 & 355 & 35.1 & 88.7 & 2165.6 & 87.8 & 500 & 12.6 & 1089 & 89 \\
\hline $18 \mathrm{R}-1,141-150$ & 242.3 & 771 & 48.0 & 0.4 & 7.8 & 741 & 0.4 & 11.9 & 443 & 331 & 31.7 & 109.6 & 2321.4 & 95.7 & 485 & 11.7 & 1102 & 76 \\
\hline $20 \mathrm{R}-1,140-150$ & 261.4 & 830 & 50.0 & 0.1 & 7.6 & 667 & 0.4 & 12.3 & 498 & 358 & 30.7 & 135.7 & 2539.5 & 104.1 & 483 & 10.7 & 1118 & 77 \\
\hline $22 \mathrm{R}-2,0-10$ & 280.8 & 884 & 58.2 & 0.3 & 7.9 & 469 & 0.4 & 14.6 & 334 & 384 & 11.5 & 227.9 & 2570.6 & 122.2 & 419 & 8.5 & 1179 & 77 \\
\hline $25 \mathrm{R}-1,95-105$ & 309.2 & 956 & 65.0 & 0.4 & 8.0 & 231 & 12.0 & 14.4 & 387 & 330 & 1.6 & 316.7 & 2337.0 & 129.1 & 329 & 5.2 & 1260 & 91 \\
\hline
\end{tabular}

tures. The subsequent decrease in their concentrations with depth probably reflects the conversion of ash into crystalline minerals during greater burial and increasing temperature and age. Lithium exhibits a behavior opposite to $\mathrm{Na}$ and $\mathrm{K}$, initially decreasing below seawater values (minimum concentration $11.8 \mu \mathrm{M}$ ), but reaching maximum concentrations $(129.1 \mu \mathrm{M})$ within the volcanic breccia units. These concentrations indicate a sedimentary sink for $\mathrm{Li}$ in the shallow sediments, but a source of $\mathrm{Li}$ in the deep sediments, probably caused by ash diagenesis and authigenesis of silicate minerals (e.g., Martin et al., 1991).

The most important hydrous mineral phases that are observed using X-ray diffraction analysis of bulk samples include clay minerals, chabazite, and analcite (Table 4) although more detailed X-ray diffractometry from Site 833 (Gerard and Person, this volume) show that other zeolite minerals are present. At shallow depths no zeolite peaks are present and clay peaks are small, but these minerals make up increasingly larger fractions of the sediment with depth (Table 4), suggesting that they are principally authigenic in origin. Although specific clay minerals have not been identified, a common, authigenic clay mineral is chlorite; its authigenesis along with analcite could cause the decrease in the pore fluid $\mathrm{Mg}$ and $\mathrm{Na}$ concentrations, respectively. Other authigenic silicate minerals could include quartz and feldspar, although quartz is only a minor component of the North Aoba Basin sediments (Table 4). Feldspars are the only crystalline phases contained in the two ash samples from Hole 833A, samples $2 \mathrm{H}-6,50-60 \mathrm{~cm}$, and $4 \mathrm{H}-6,60-70 \mathrm{~cm}$ (Table 4). The proportion of detrital to authigenic feldspars is unknown, but feldspars dominate the bulk sediment at shallow depths where pore-fluid composition is 
Table 1 (continued).

\begin{tabular}{|c|c|c|c|c|c|c|c|c|c|c|c|c|c|c|c|c|c|c|}
\hline $\begin{array}{l}\text { Core, section, } \\
\text { interval }(\mathrm{cm})\end{array}$ & $\begin{array}{l}\text { Depth } \\
\text { (mbsf) }\end{array}$ & $\underset{(\mathrm{mM})}{\mathrm{Cl}}$ & Salinity & $\begin{array}{c}\text { Alkalinity } \\
(\mathrm{mM})\end{array}$ & $\mathrm{pH}$ & $\begin{array}{l}\mathrm{NH}_{4} \\
(\mu \mathrm{M})\end{array}$ & $\begin{array}{l}\mathrm{PO}_{4} \\
(\mu \mathrm{M})\end{array}$ & $\begin{array}{c}\mathrm{SO}_{4} \\
(\mathrm{mM})\end{array}$ & $\begin{array}{c}\mathrm{Si} \\
(\mu \mathrm{M})\end{array}$ & $\begin{array}{c}\text { B } \\
(\mu \mathrm{M})\end{array}$ & $\begin{array}{c}\mathrm{Mg} \\
(\mathrm{mM})\end{array}$ & $\begin{array}{c}\mathrm{Ca} \\
(\mathrm{mM})\end{array}$ & $\begin{array}{c}\mathrm{Sr} \\
(\mu \mathrm{M})\end{array}$ & $\begin{array}{c}\mathrm{Li} \\
(\mu \mathrm{M})\end{array}$ & $\begin{array}{c}\mathrm{Na} \\
(\mathrm{mM})\end{array}$ & $\underset{(\mathrm{mM})}{\mathrm{K}}$ & $\begin{array}{c}\mathrm{Br} \\
(\mu \mathrm{M})\end{array}$ & $\begin{array}{c}\mathrm{I} \\
(\mu \mathrm{M})\end{array}$ \\
\hline \multicolumn{19}{|l|}{ 134-833B- } \\
\hline $27 \mathrm{R}-1,136-150$ & 328.9 & 1014 & 70.0 & 0.3 & 8.0 & 43 & 0.6 & 13.1 & 378 & 368 & 0.7 & 373.5 & 1651.5 & 127.6 & 284 & 3.2 & 1350 & 96 \\
\hline $29 \mathrm{R}-1,117-131$ & 348.1 & 1042 & 72.0 & 0.5 & 8.4 & 48 & 0.4 & 13.9 & 414 & 436 & 0.0 & 399.5 & 950.5 & 115.4 & 237 & 3.6 & 1380 & 104 \\
\hline $31 \mathrm{R}-2,135-150$ & 369.1 & 1015 & 72.0 & 0.3 & 8.0 & 48 & 0.6 & 13.3 & 382 & 344 & 0.0 & 389.0 & 997.2 & 116.6 & 242 & 2.5 & 1345 & 100 \\
\hline $33 \mathrm{R}-1,112-125$ & 386.3 & 988 & 69.5 & 0.4 & 8.5 & 0 & 0.4 & 12.2 & 85 & 357 & 0.0 & 381.0 & 374.1 & 97.0 & 228 & 1.5 & 1367 & 99 \\
\hline $38 \mathrm{R}-4,135-150$ & 439.3 & 1141 & 81.5 & & & & & 12.7 & & & & 491.6 & & & 187 & 0.7 & & \\
\hline $46 \mathrm{R}-3,135-150$ & 494.5 & 1241 & 88.3 & 0.5 & 8.6 & 0 & 0.4 & 12.0 & 160 & 521 & 0.0 & 548.5 & 132.7 & 118.1 & 197 & 0.7 & 1574 & 110 \\
\hline $55 R-4,10-30$ & 583.8 & 960 & 71.0 & & & & & 10.4 & & & & 407.6 & & & 163 & 0.4 & & \\
\hline $60 \mathrm{R}-5,0-18$ & 631.4 & 908 & 65.0 & & & & & 5.4 & & 404 & & 385.2 & 131.8 & 96.4 & 150 & 0.8 & 1272 & 152 \\
\hline $63 \mathrm{R}-2,0-15$ & 656.4 & 851 & 60.0 & 0.2 & 8.0 & 0 & 0.4 & 6.2 & 173 & 427 & 8.1 & 349.4 & 67.8 & 88.3 & 166 & 1.3 & 1240 & 135 \\
\hline $66 R-4,0-15$ & 688.3 & 828 & 59.0 & & & 0 & 0.4 & 5.6 & 137 & 319 & 4.4 & 336.9 & 185.3 & 85.7 & 168 & 0.8 & 1141 & 136 \\
\hline $69 \mathrm{R}-2,0-15$ & 713.7 & 805 & 57.0 & & & & & 6.0 & & & & 299.3 & & & 186 & 0.7 & & \\
\hline $74 R-2,130-150$ & 763.6 & 719 & 57.5 & & & & & 7.3 & 130 & & & 213.3 & & & 276 & 1.3 & & \\
\hline \multicolumn{19}{|l|}{$134-827 \mathrm{~A}$ - } \\
\hline $1 \mathrm{H}-4,145-150$ & 6.0 & 561 & 38.5 & 18.2 & 7.9 & 789 & 3.3 & 30.5 & 349 & 448 & 60.9 & 11.7 & 53.8 & 6.8 & 488 & 11.8 & 833 & 170 \\
\hline $3 \mathrm{H}-4,145-150$ & 25.3 & 580 & 36.0 & 15.0 & 7.8 & 1441 & 6.6 & 24.1 & 298 & 296 & 71.7 & 9.8 & 92.1 & 4.7 & 468 & 10.3 & 846 & 391 \\
\hline $6 \mathrm{H}-4,145-150$ & 50.9 & 598 & 35.0 & 23.6 & 8.0 & 1925 & 18.2 & 0.0 & 352 & 350 & 66.0 & 7.0 & 89.6 & 3.3 & 455 & 11.4 & 893 & 400 \\
\hline $9 \mathrm{H}-2,145-150$ & 70.0 & 596 & 35.5 & 18.7 & 7.9 & 1839 & 7.1 & 0.0 & 543 & 401 & 59.4 & 10.3 & 93.0 & 4.3 & 474 & 10.6 & 892 & 240 \\
\hline $13 \mathrm{H}-2,135-140$ & 90.8 & 600 & 35.0 & 2.3 & 8.1 & 1337 & 1.9 & 1.0 & 356 & 378 & 41.0 & 21.4 & 117.6 & 14.6 & 475 & 13.2 & 897 & 122 \\
\hline \multicolumn{19}{|l|}{$134-827 \mathrm{~B}-$} \\
\hline $2 \mathrm{R}-3,145-150$ & 122.1 & 604 & 38.5 & & 8.0 & 1400 & 1.3 & 5.7 & 459 & 294 & 36.7 & 49.6 & 165.3 & 18.8 & 434 & 12,9 & 897 & 89 \\
\hline $5 R-2,140-150$ & 149.5 & 614 & 38.0 & 0.4 & 7.3 & 835 & 0.6 & 6.7 & 511 & 252 & 29.2 & 99.4 & 179.7 & 34.8 & 359 & 12.5 & 888 & 81 \\
\hline $8 R-4,140-150$ & 181.4 & 628 & 40.0 & & & 691 & & 3.3 & 453 & & 13.2 & 157.0 & & & 277 & 10.9 & & \\
\hline $11 \mathrm{R}-1,135-150$ & 205.9 & 622 & 40.0 & 0.1 & 6.8 & 841 & & 3.2 & 356 & & 9.3 & 175.8 & & & 248 & 9.0 & & \\
\hline $14 \mathrm{R}-2,0-30$ & 236.3 & 606 & 40.0 & 0.5 & 8.3 & 454 & 0.6 & 8.7 & 270 & 286 & 9.8 & 172.3 & 126.2 & 47.1 & 253 & 6.3 & 898 & 52 \\
\hline \multicolumn{19}{|l|}{$134-829 \mathrm{~A}-$} \\
\hline IR-1, 146-150 & 1.5 & 558 & 35.0 & 5.9 & 7.8 & 246 & 13.2 & 26.6 & 422 & 421 & 45.0 & 9.3 & 82.9 & 8.2 & 487 & 11.3 & 860 & 21 \\
\hline $3 \mathrm{R}-2,145-150$ & 15.3 & 561 & 35.5 & 9.0 & 8.0 & 676 & 12.9 & 20.0 & 450 & 397 & 42.2 & 8.9 & 82.9 & 5.7 & 488 & 11.6 & 861 & 36 \\
\hline $5 \mathrm{R}-5,145-150$ & 38.5 & 561 & 34.0 & 5.0 & 7.8 & 729 & 5.8 & 19.6 & 543 & 441 & 38.0 & 11.1 & 150.8 & 9.3 & 490 & 10.7 & 874 & 48 \\
\hline $7 \mathrm{R}-1,145-150$ & 52.3 & 562 & 34.0 & 2.2 & 7.8 & 459 & 1.3 & 23.4 & 501 & 441 & 34.6 & 14.6 & 396.2 & 19.3 & 485 & 10.6 & 878 & 45 \\
\hline $12 \mathrm{R}-5,140-150$ & 104.9 & 564 & 32,5 & 14.3 & 8.0 & 1319 & 24.4 & 0.6 & 531 & 374 & 38.9 & 4.8 & 75.5 & 5.5 & 477 & 10.4 & 876 & 95 \\
\hline $14 \mathrm{R}-4,135-150$ & 124.1 & 568 & 32.5 & 12.0 & 8.0 & 1482 & 24.3 & 3.5 & 475 & 347 & 37.2 & 5.0 & 68.8 & 4.7 & 482 & 10.3 & 860 & 83 \\
\hline $16 R-4,135-150$ & 143.4 & 565 & 33.0 & 7.7 & 8.1 & 1521 & 11.0 & 10.6 & 527 & 362 & 35.8 & 7.3 & 108.6 & 5.9 & 482 & 11.0 & 870 & 64 \\
\hline $18 \mathrm{R}-3,135-150$ & 161.2 & 564 & 34.0 & 4.4 & 8.2 & 1163 & & 17.2 & 482 & 375 & 34.4 & 12.3 & 213.7 & 9.3 & 479 & 10.6 & 849 & 53 \\
\hline $20 \mathrm{R}-2,130-150$ & 178.9 & 565 & & & & 674 & & 23.3 & 283 & 360 & 38.2 & 15.6 & 440.7 & 16.9 & 470 & 10.1 & 867 & 37 \\
\hline $44 \mathrm{R}-1,123-140$ & 417.8 & 424 & & & & & & 12.2 & & & 16.5 & & & & 396 & 7.7 & & \\
\hline $56 \mathrm{R}-3,130-150$ & 517.7 & 709 & & & & & & 4.5 & & & 20.2 & & 61.0 & & 362 & 7.2 & 636 & \\
\hline $57 \mathrm{R}-2,0-33$ & 515.0 & 417 & & & & & & 7.3 & & & 21.5 & & 75.4 & & 365 & 7.6 & 653 & \\
\hline \multicolumn{19}{|l|}{$134-829 \mathrm{~B}-$} \\
\hline $2 \mathrm{H}-2,145-150$ & 3.5 & 554 & 34.5 & 6.6 & 7.9 & 403 & 16.1 & 18.8 & 334 & 456 & 42.6 & 9.2 & 81.3 & 4.9 & 470 & 11.3 & & 32 \\
\hline \multicolumn{19}{|l|}{$134-829 \mathrm{C}-$} \\
\hline IH-2, 145-150 & 3.0 & 556 & 35.0 & 6.8 & 7.9 & 417 & 12.2 & 22.2 & 341 & 424 & 42.9 & 9.0 & 84 & 4.9 & 472 & 11.7 & & 23 \\
\hline $1 \mathrm{H}-4,145-150$ & 6.0 & 556 & 35.0 & & 7.9 & 781 & 17.0 & 17.0 & 416 & 416 & 42.7 & 8.6 & 82.9 & 4.7 & 484 & 10.5 & 856 & 35 \\
\hline $2 \mathrm{H}-$ & 9.8 & 556 & 34.5 & & 7.9 & 987 & 20.3 & 13.0 & 440 & 389 & 41.1 & 7.8 & 82.5 & 5.0 & 484 & 10.4 & 860 & 46 \\
\hline $3 \mathrm{H}-6,135-140$ & 26.8 & 558 & 34.0 & 7.7 & 7.8 & 907 & 12.9 & 13.3 & 458 & 412 & 36.2 & 8.4 & 94.5 & 6.5 & 472 & 11.6 & 865 & 58 \\
\hline \multicolumn{19}{|l|}{$134-830 \mathrm{~A}-$} \\
\hline $1 \mathrm{H}-3,145-150$ & 4.5 & 552 & 35.0 & 3.7 & 7.9 & 216 & 4.8 & 22.8 & 456 & 338 & 46.4 & 8.4 & 85.3 & 16.8 & 469 & 12.0 & 860 & 17 \\
\hline $145-150$ & 22.5 & 564 & 32.0 & 2.7 & 7.8 & 605 & 1.8 & 4.9 & 319 & 415 & 38.1 & 8.2 & 91.3 & 7.5 & 467 & 10.6 & 876 & 55 \\
\hline $5 \mathrm{H}-1,145-150$ & 36.4 & 568 & 32.0 & 2.5 & 8.1 & 952 & 1.8 & 4.1 & 319 & 313 & 35.8 & 10.3 & 103.2 & 6.5 & 456 & 9.1 & 894 & 81 \\
\hline \multicolumn{19}{|l|}{ 134-830B- } \\
\hline $.140-150$ & 59.5 & 575 & 32.0 & & & 1518 & 0.9 & 1.3 & 300 & 290 & 33.8 & 21.7 & 118.5 & 9.8 & 448 & 6.4 & 912 & 140 \\
\hline $100-110$ & 78.5 & 57 & 32 & 1.2 & 8.2 & 1570 & 1.0 & 0.5 & 314 & 303 & 34.7 & 25 . & 127.9 & 9.8 & 451 & 7.6 & 911 & 172 \\
\hline $6 \mathrm{R}-1,134-150$ & 98.4 & 578 & 33.5 & 1.4 & 8.1 & 1547 & 0.8 & 1.4 & 338 & 349 & 38.3 & 24.8 & 125.3 & 10.5 & 439 & 7.3 & 931 & 183 \\
\hline $8 \mathrm{R}-1,134-150$ & 117.8 & 584 & 34.0 & 1.7 & 8.0 & 1786 & 0.9 & 1.9 & 456 & 276 & 41.6 & 23.4 & 125.3 & 10.3 & 436 & 9.1 & 959 & 185 \\
\hline $10 \mathrm{R}-2,0-19$ & 141.0 & 587 & 34.0 & 1.5 & 8.1 & 1849 & 0.9 & 1.7 & 453 & 267 & 41.7 & 28.9 & 142.3 & 14.1 & 429 & 10.0 & 922 & 200 \\
\hline $12 \mathrm{R}-2,0-18$ & 156.8 & 597 & 34.0 & 1.3 & 7.9 & 1629 & 0.6 & 2.5 & 488 & 292 & 36.8 & 57.9 & 160.2 & 22.6 & 403 & 9.9 & 975 & 190 \\
\hline $18 \mathrm{R}-1,135-155$ & 214.8 & 620 & 43.5 & & & 0 & 0.5 & 14.7 & 153 & 168 & 3.5 & 208.2 & 138.1 & 66.3 & 216 & 0.0 & 952 & 134 \\
\hline \multirow{2}{*}{\multicolumn{19}{|c|}{$\begin{array}{l}134-830 \mathrm{C}- \\
10 \mathrm{R}-2,0-10\end{array}$}} \\
\hline $10 \mathrm{R}-2,0-10$ & 323.0 & 603 & 42.5 & & & & & & & & & & & & & & & \\
\hline $12 \mathrm{R}-2,28-53$ & 341.2 & 613 & 42.0 & 0.4 & 8.1 & 5 & 0.5 & 9.5 & 137 & & 0.0 & 229.2 & 58.9 & 58.9 & 172 & 0.0 & 943 & 85 \\
\hline
\end{tabular}

not greatly altered from seawater value, suggesting that much of the feldspar is detrital.

The modern temperature gradients in the North Aoba Basin are $42^{\circ} \mathrm{C} / \mathrm{km}$ at Site 832 and $67^{\circ} \mathrm{C} / \mathrm{km}$ at Site 833 (Fig. 6A). Based on these gradients, and a bottom-water temperature of $3^{\circ} \mathrm{C}$, Units II and IV at Site 832 reach temperatures of approximately $19^{\circ} \mathrm{C}$ and $25^{\circ} \mathrm{C}$, respectively, and Unit III at Site 833 reaches a temperature of $34^{\circ} \mathrm{C}$. Higher temperatures are probably responsible for more extensive diagenesis and thus the greater changes in pore-fluid composition at Site 833 than Site 832 , but even the higher temperatures are lower than commonly assigned to metamorphic facies based on such authigenic mineral assemblages. Although the heat flow and geothermal gradients would have been greater during times of intrusion of the sills found at the base of Site 833 (Fig. 2), the modern pore fluid concentrations imply that the authigenic minerals are currently forming, even at these low formation temperatures. The sills were intruded at $3.3 \pm 0.3$ and $3.65 \pm 0.18 \mathrm{Ma}$ (Rex, this volume) under approximately $110 \mathrm{~m}$ of overlying sediment cover. Assuming an emplacement temperature of $\sim 1500^{\circ} \mathrm{C}$ and based on thermodynamic data given in Robie et al. (1979) and the measured thermal conductivities (Collot, Greene, Stokking, et al., 1992), simple diffusion would have dissipated heat from the sill in $<10^{4}$ years. All sediment younger than 3.3 m.y. (shallower than $\sim 700 \mathrm{mbsf}$ ) would be unaffected by this heat. In the absence of additional heat sources, the authigenic minerals would have formed at the relatively cool temperatures governed by temperature gradients similar to the modern ones (Fig. 6A).

An approximately linear exchange of $\mathrm{Ca}$ for $\mathrm{Na}, \mathrm{K}$, and $\mathrm{Mg}$ occurs in the pore fluids (Fig. 7A), but the slope of the line is not unity 
Table 2. Stable isotope ratios of water.

\begin{tabular}{|c|c|c|c|}
\hline $\begin{array}{l}\text { Core, section, } \\
\text { interval }(\mathrm{cm})\end{array}$ & $\begin{array}{l}\text { Depth } \\
\text { (mbsf) }\end{array}$ & $\begin{array}{l}\delta^{18} \mathrm{O} \\
(\%)\end{array}$ & $\begin{array}{l}\delta \mathrm{D} \\
(\%)\end{array}$ \\
\hline \multicolumn{4}{|l|}{$134-827 \mathrm{~A}$ - } \\
\hline $1 \mathrm{H}-4,145-150$ & 6.0 & -0.5 & -5.0 \\
\hline $6 \mathrm{H}-4,145-150$ & 50.9 & -1.6 & +3.0 \\
\hline $13 \mathrm{H}-2,135-140$ & 90.8 & -2.9 & -3.0 \\
\hline \multicolumn{4}{|l|}{$143-827 \mathrm{~B}-$} \\
\hline SR-2, 140-150 & 149.5 & -4.2 & -1.5 \\
\hline $14 \mathrm{R}-2,0-30$ & 236.3 & -5.0 & +3.0 \\
\hline \multicolumn{4}{|l|}{$134-829 \mathrm{~A}-$} \\
\hline 1R-1, 146-150 & 1.5 & -0.2 & +2.5 \\
\hline 5R-5, $145-150$ & 38.5 & -3.0 & -1.0 \\
\hline $7 \mathrm{R}-1,145-150$ & 52.3 & -0.7 & +5.5 \\
\hline $12 \mathrm{R}-4,140-150$ & 104.9 & +0.5 & -1.5 \\
\hline $16 \mathrm{R}-4,135-150$ & 143.4 & -0.7 & +1.5 \\
\hline $20 \mathrm{R}-2,130-150$ & 178.9 & -0.8 & -3.0 \\
\hline $56 \mathrm{R}-3,130-150$ & 508.1 & -0.7 & -8.0 \\
\hline $57 \mathrm{R}-2,0-33$ & 515.0 & -0.4 & -5.0 \\
\hline \multicolumn{4}{|l|}{$134-833 \mathrm{~A}-$} \\
\hline $1 \mathrm{H}-3,145-150$ & 4.5 & -0.9 & -4.5 \\
\hline $3 \mathrm{H}-3,145-150$ & 23.5 & -2.2 & +2.5 \\
\hline $8 \mathrm{H}-1,145-150$ & 55.5 & -2.7 & +7.5 \\
\hline $16 X-2,140-150$ & 95.6 & -3.5 & +2.5 \\
\hline $21 X-2,140-150$ & 144.9 & -4.2 & -0.5 \\
\hline \multicolumn{4}{|l|}{ 134-833B- } \\
\hline $16 \mathrm{R}-1,140-150$ & 223.5 & -5.2 & +2.5 \\
\hline $22 \mathrm{R}-2,0-10$ & 280.8 & -5.9 & +0.5 \\
\hline $33 R-1,112-125$ & 386.3 & -7.1 & +2.5 \\
\hline $46 \mathrm{R}-3,135-150$ & 494.5 & -9.5 & +8.5 \\
\hline $63 R-2,0-15$ & 656.4 & -6.8 & +2.0 \\
\hline $66 \mathrm{R}-4,0-15$ & 688.3 & -6.1 & -2.5 \\
\hline
\end{tabular}

Table 3. Strontium isotope ratios of dissolved $\mathrm{Sr}$.

\begin{tabular}{|c|c|c|c|}
\hline $\begin{array}{l}\text { Core, section, } \\
\text { interval }(\mathrm{cm})\end{array}$ & $\begin{array}{l}\text { Depth } \\
\text { (mbsf) }\end{array}$ & $\begin{array}{c}\mathrm{Sr} \\
(\mathrm{mM})\end{array}$ & ${ }^{87} \mathrm{Sr} /{ }^{86} \mathrm{~S}$ \\
\hline \multicolumn{4}{|l|}{$134-827 \mathrm{~A}=$} \\
\hline $1 \mathrm{H}-4,145-150$ & 6.0 & 53.8 & 0.7084 \\
\hline $6 \mathrm{H}-4,145-150$ & 50.9 & 89.6 & \\
\hline $13 \mathrm{H}-2,135-140$ & 90.8 & 117.6 & 0.70882 \\
\hline \multicolumn{4}{|l|}{ 134-827B- } \\
\hline SR-2, $140-150$ & 149.5 & 179.7 & 0.70855 \\
\hline $14 \mathrm{R}-2,0-3$ & & 126.2 & \\
\hline \multicolumn{4}{|l|}{$134-829 \mathrm{~A}$ - } \\
\hline $1 \mathrm{R}-1,146$ & 1.5 & 82.9 & 0.70874 \\
\hline $5 R-5,1$ & 38.5 & 150.8 & 0.70868 \\
\hline $7 \mathrm{R}-1,145$ & 52.3 & 396.2 & 0.7 \\
\hline $12 R-5,140-150$ & 104.9 & 75.5 & 0.708 \\
\hline $16 \mathrm{R}-4,135-150$ & 143.4 & 108.6 & 0.70853 \\
\hline $30-150$ & 178.9 & 440.7 & 0.7084 \\
\hline 150 & 517.7 & 61.0 & \\
\hline $57 \mathrm{R}-2,0-3$ & 515.0 & 75.4 & 0.70 \\
\hline \multicolumn{4}{|l|}{$134-833 \mathrm{~A}-$} \\
\hline $1 \mathrm{H}-3,145$ & 4.5 & 69.6 & 0.708403 \\
\hline $3 \mathrm{H}$ & 23.5 & 65.3 & 0.707871 \\
\hline $145-150$ & 55.5 & 68.8 & 0.707 \\
\hline $16 X-2,140-150$ & 95.6 & 135.2 & 0.7068 \\
\hline $21 X-2,140-1$ & 144.9 & 219.9 & 0.70728 \\
\hline \multicolumn{4}{|l|}{ 134-833B- } \\
\hline $16 \mathrm{R}-1,14$ & 223.5 & 2165.6 & 0.708 \\
\hline $22 \mathrm{R}-2,0-1$ & 280.8 & 2570.6 & 0.70865 \\
\hline $33 \mathrm{R}-1,112-1$ & 386.3 & 374.1 & 0.70781 \\
\hline $46 \mathrm{R}-3,135-150$ & 494.5 & 132.7 & 0.70606 \\
\hline $66 \mathrm{R}-4,0-15$ & 688.3 & 185.3 & 0.70673 \\
\hline
\end{tabular}

Note: All errors less than standard error except for Sample $833 \mathrm{~A}-1 \mathrm{H}-3$, which has an error of .000026 .

indicating that this exchange does not maintain the charge balance. The balance is maintained by changes in anion concentrations, principally $\mathrm{Cl}$, although changes in the $\mathrm{SO}_{4}$ concentration also are important (Fig. 7B). The changes in $\mathrm{Cl}$ concentration are extreme, reaching maxima of $742 \mathrm{mM}$ at Site 832 and $1241 \mathrm{mM}$ at Site 833 (Table 1). Other than during reactions with evaporite minerals such as halite, $\mathrm{Cl}$
Table 4. Bulk sediment $\mathrm{X}$-ray diffraction results.

\begin{tabular}{|c|c|c|}
\hline $\begin{array}{l}\text { Core, section, } \\
\text { interval }(\mathrm{cm})\end{array}$ & $\begin{array}{l}\text { Depth } \\
\text { (mbsf) }\end{array}$ & Major Mineralogy \\
\hline \multicolumn{3}{|l|}{$134-829 \mathrm{~A}-$} \\
\hline $44 \mathrm{R}-1,123-140$ & 418 & Calcite $\gg$ Feldspars $\gg$ Quartz \\
\hline $56 \mathrm{R}-3,130-150$ & 508 & Calcite $>$ Feldspars $\geq$ Clays \\
\hline $57 \mathrm{R}-2,0-33$ & 515 & Calcite $\gg$ Feldspars $\geq$ Clays \\
\hline \multicolumn{3}{|l|}{$134-832 \mathrm{~A}-$} \\
\hline $2 \mathrm{H}-6,50-60$ & 8 & Feldspars \\
\hline $4 \mathrm{H}-6,60-70$ & 27 & Feldspars \\
\hline \multicolumn{3}{|l|}{ 134-832B- } \\
\hline $15 \mathrm{R}-2,58-68$ & 280 & Calcite $>$ Feldspars $>$ Quartz \\
\hline $21 R-3,140-150$ & 342 & Feldspars $>$ Calcite $>$ Clays \\
\hline $28 \mathrm{R}-2,135-150$ & 408 & Feldspars $>$ Chabazite $\geq$ Clays \\
\hline $30 R-4,138-150$ & 428 & Calcite $>$ Feldspars $>$ Clays \\
\hline $32 \mathrm{R}-2,135-150$ & 446 & Calcite >Feldspars \\
\hline $34 \mathrm{R}-1,135-150$ & 463 & Calcite $\simeq$ Feldspars \\
\hline $40 \mathrm{R}-1,123-135$ & 521 & Calcite $>$ Feldspars $\gg$ Clays \\
\hline \multicolumn{3}{|l|}{ 134-833B- } \\
\hline $16 \mathrm{R}-1,140-150$ & 224 & Calcite $\simeq$ Feldspars $\gg$ Clays \\
\hline $22 \mathrm{R}-2,0-10$ & 281 & Calcite $>$ Feldspars $\simeq$ Clays \\
\hline $27 \mathrm{R}-1,136-150$ & 329 & Calcite $\geq$ Feldspars $>$ Clays \\
\hline $33 \mathrm{R}-1,112-125$ & 386 & Analcite $>$ Calcite $>$ Clays $\simeq$ Feldspars \\
\hline $38 \mathrm{R}-1,135-150$ & 439 & Feldspars $\gg$ Calcite \\
\hline $46 \mathrm{R}-3,135-150$ & 495 & Calcite $>$ Analcite $>$ Feldspars $\geq$ Clays \\
\hline $55 \mathrm{R}-4,10-30$ & 584 & Calcite $>$ Analcite $>$ Clays $\simeq$ Feldspars \\
\hline $66 \mathrm{R}-4,0-15$ & 688 & Analcite $>$ Calcite $>$ Clays $>$ Feldspars \\
\hline $74 \mathrm{R}-2,130-150$ & 764 & Calcite $>$ Analcite $>$ Clays $>$ Feldspars \\
\hline
\end{tabular}

Note: Mineralogy is based on relative peak areas. No correction factors have been applied.

should behave conservatively, in all common diagenetic reactions within marine sediments. In organic matter-poor sediments, $\mathrm{Br}$ also behaves conservatively, and thus during processes such as the early stages of seawater evaporation, the $\mathrm{Br} / \mathrm{Cl}$ molar ratio should not change from the seawater value of $1.54 \times 10^{-3}$. The observed molar ratio of $1.05 \times 10^{-3}$ (Fig. $8 \mathrm{~A}$ ) indicates that the high $\mathrm{Cl}$ and $\mathrm{Br}$ concentrations do not result from evaporation and that one or both of the ions are not conservative. $\mathrm{The} \mathrm{Br} / \mathrm{Cl}$ ratio is greater than expected from halite dissolution (e.g., Holser, 1966, 1970, 1979; Holser and Wilgus, 1981) and no evaporites have been observed at the New Hebrides Island Arc, indicating that evaporitic dissolution does not cause the high $\mathrm{Cl}$ concentrations. Alternatively, the high $\mathrm{Cl}$ and $\mathrm{Br}$ concentrations could be caused by removal of $\mathrm{H}_{2} \mathrm{O}$ from the pore fluid during authigenesis of hydrous minerals. The conversion of ash into hydrous minerals could also alter the $\mathrm{Br} / \mathrm{Cl}$ ratio depending on the unknown $\mathrm{Br} / \mathrm{Cl}$ ratio in the ash, or by differences in distribution of $\mathrm{Br}$ and $\mathrm{Cl}$ into the authigenic minerals. $\mathrm{Br} / \mathrm{Cl}$ molar ratios in typical andesitic lavas range from 1.5 to $6.3 \times 10^{-3}$ (Gill, 1981, and references therein), considerably greater than seawater value, and should thus increase the pore-fluid $\mathrm{Br} / \mathrm{Cl}$ ratio during dissolution. The $\mathrm{Br} / \mathrm{Cl}$ ratio would decrease if $\mathrm{Br}$ has a greater distribution than $\mathrm{Cl}$ into authigenic minerals and much water is incorporated in the hydrous minerals as $\mathrm{OH}^{-}$, likely structural sites for both $\mathrm{Br}^{-}$and $\mathrm{Cl}^{-}$. Because these ions have the same valence, their distributions would be controlled by size differences.

Assuming that the $\mathrm{Cl}$ concentrations increase solely from the transfer of water from the pore fluids into the authigenic, hydrous minerals, the mass of water, $M_{w}$, transferred per gram of sediment is

$$
M_{w}=\left(1-R_{C l}\right) \cdot R_{w c}
$$

where $\mathrm{R}_{\mathrm{Cl}}$ is the ratio of seawater $\mathrm{Cl}$ concentration to the measured pore-fluid concentration and $\mathrm{R}_{\mathrm{wc}}$ is the ratio of the mass of pore water to the mass of the dry sediment taken from Collot, Greene, Stokking, et al. (1992). These calculations indicate that up to $0.2 \mathrm{~g}$ of water is incorporated per gram of sediment (i.e., $20 \mathrm{wt} \%$ ). Because the $\mathrm{Br} / \mathrm{Cl}$ 

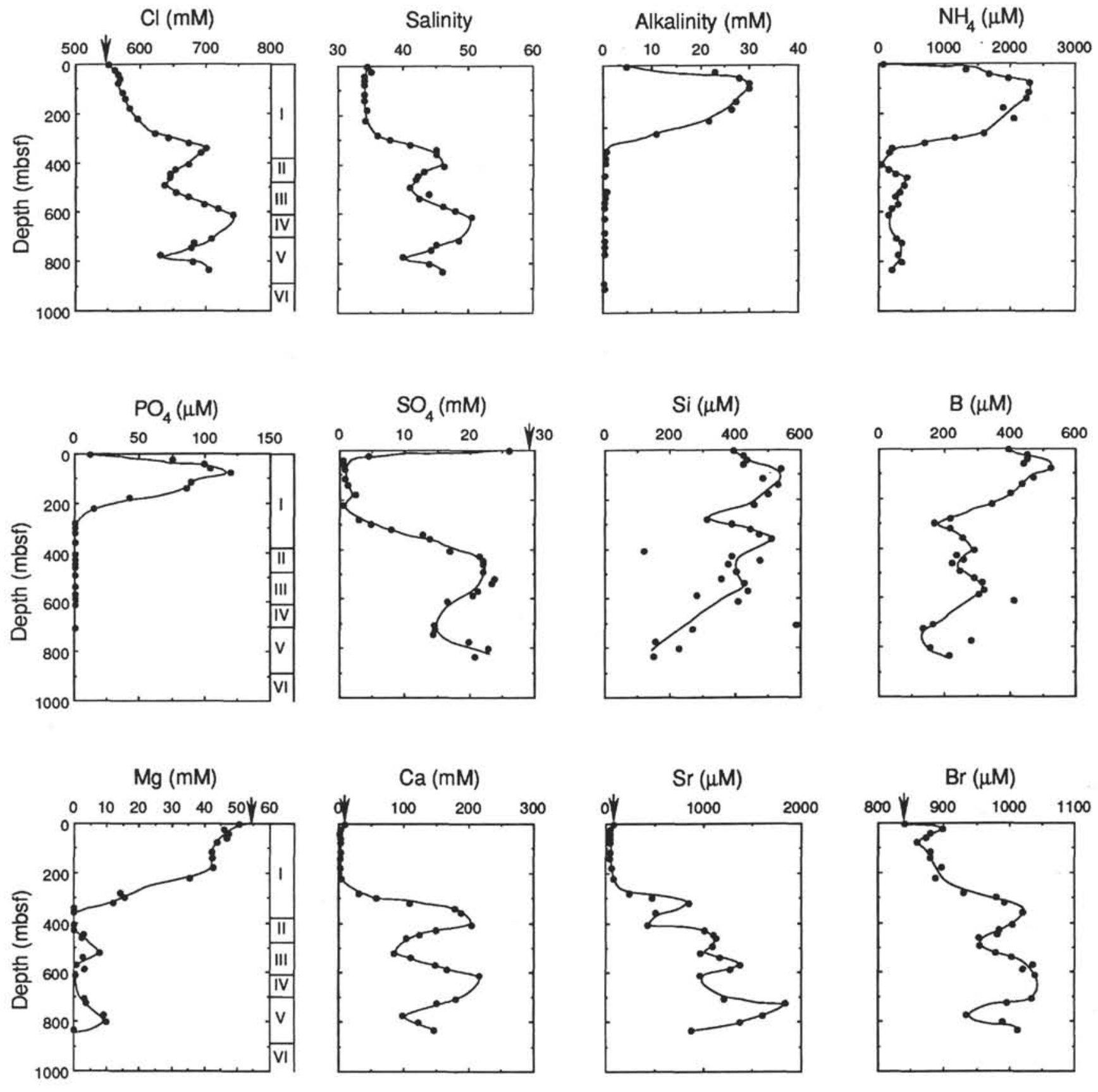

$\mathrm{Br}(\mu \mathrm{M})$
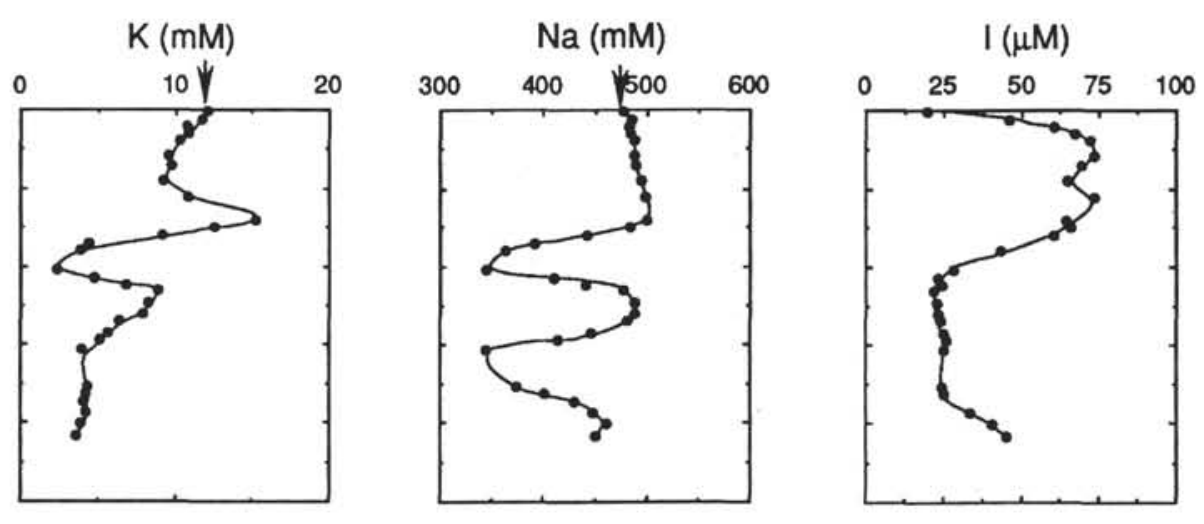

Figure 4. Depth profiles of pore fluid species and unit thicknesses for North Aoba Basin Site 832. Arrows indicate seawater composition. 

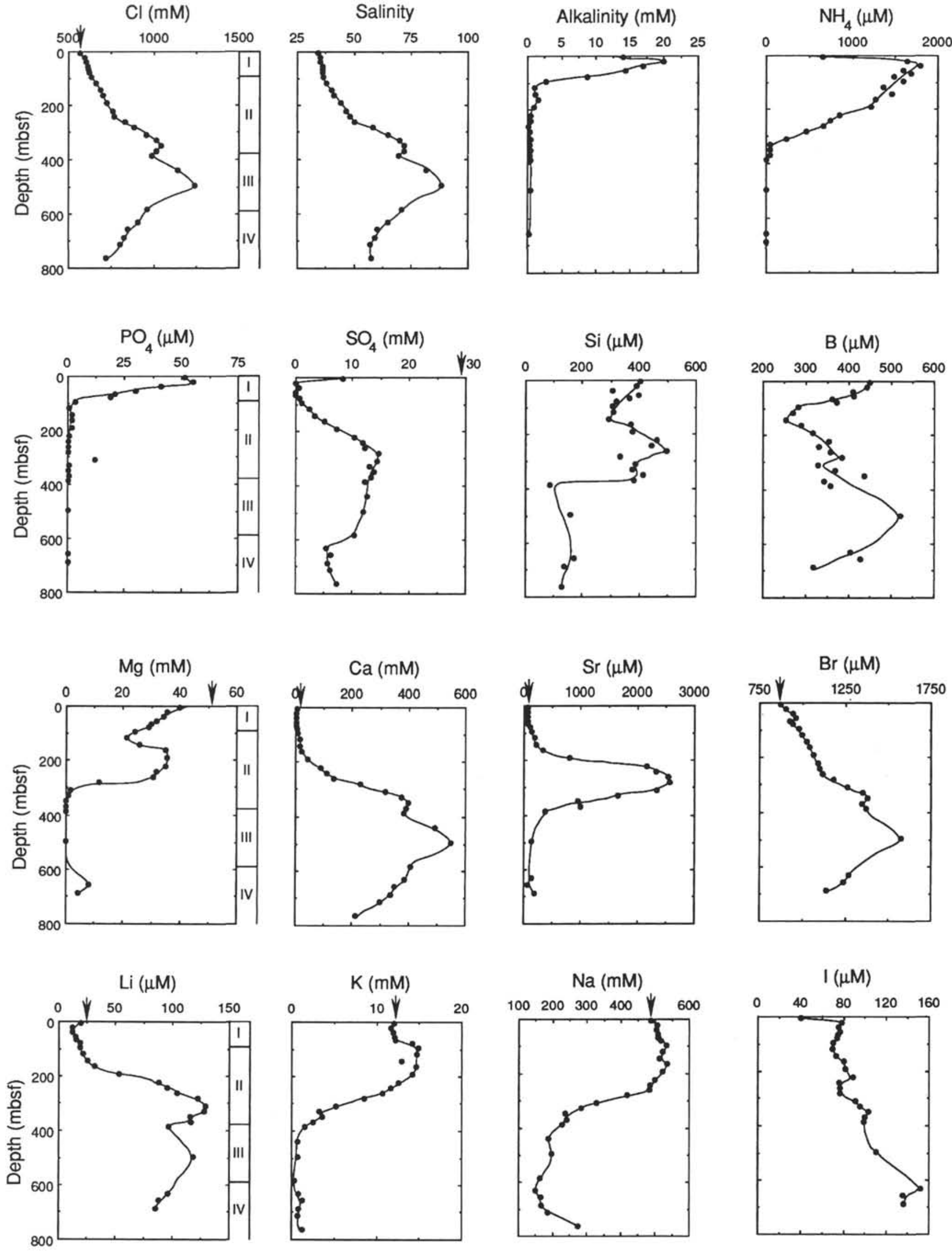

$\mathrm{Na}(\mathrm{mM})$

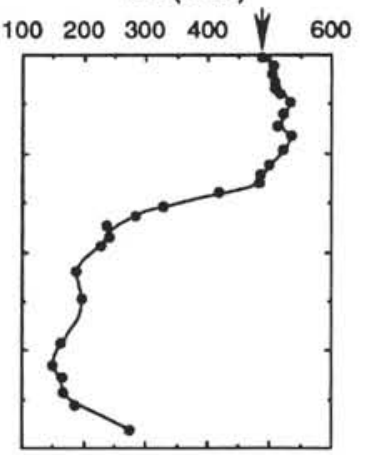

I ( $\mu \mathrm{M})$

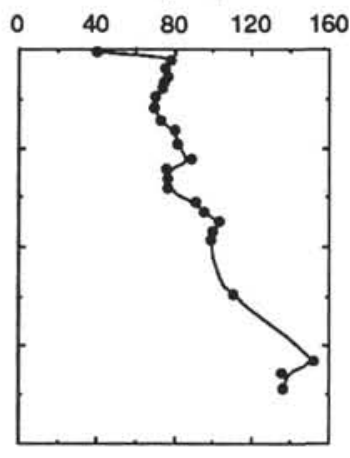

Figure 5. Depth profiles of pore fluid species and unit thicknesses for North Aoba Basin Site 833. Arrows indicate seawater composition. 

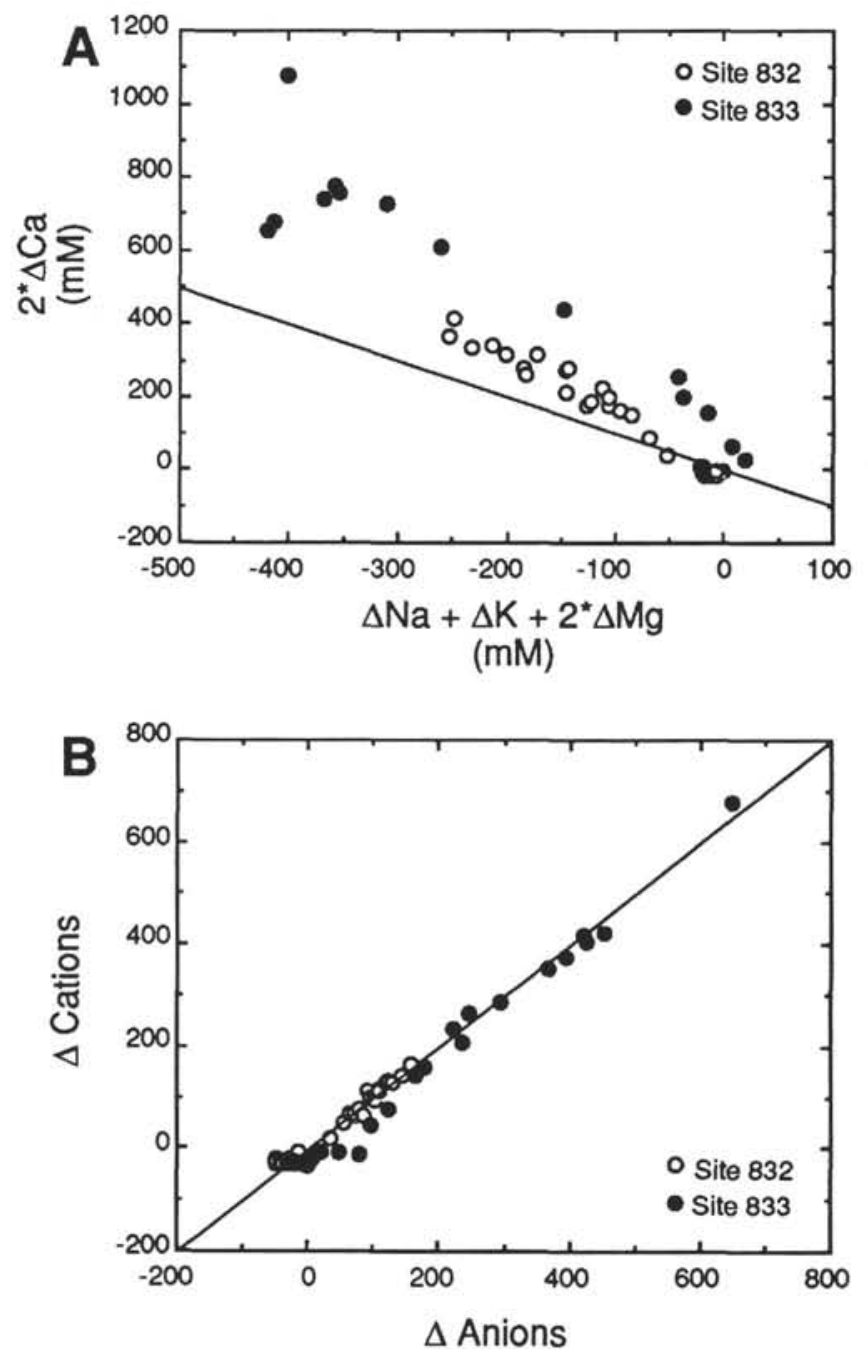

Figure 6. A. Plot of $2 \Delta \mathrm{Ca}$ vs. sum of $\Delta \mathrm{Na}+\Delta \mathrm{K}+2 \cdot \Delta \mathrm{Mg}$ for the North Aoba Basin Sites 832 and 833 . The $\Delta$ indicates the difference between the measured and seawater cation concentrations. The solid line indicates a slope of -1 . B. Plot of the difference between the sum of the measured $\mathrm{Na}, \mathrm{K}, \mathrm{Mg}$, and $\mathrm{Ca}$ concentrations and seawater concentrations vs. the difference between the sum of the $\mathrm{Cl}$ and $\mathrm{SO}_{4}$ concentrations and seawater concentrations. The solid line indicates a slope of 1 .

ratio is $\sim 32 \%$ lower than seawater value, a similar calculation based on $\mathrm{Br}$ concentration would indicate less water is incorporated into the sediments. The total water in clays and zeolites, including interlayer, channel and structural, ranges from 8 to $25 \mathrm{wt} \%$ (Sand and Mumpton, 1976; Newman, 1987), where the highest value is for smectite with three interlayer water molecules. The water contained in pure smectite is only slightly greater than the maximum value calculated from Equation 1, and therefore authigenesis of a pure smectite layer would be required to produce the maximum calculated level of hydration. Because the mineralogy in the North Aoba Basin is a mixture of clay and other minerals (Table 4), this calculated mass of water transferred is not supported by the mineralogy. In addition, because some $\mathrm{Cl}$ will diffuse from regions with maximum $\mathrm{Cl}$ concentrations and some $\mathrm{Cl}$ will be lost from the pore fluid due to its distribution into authigenic minerals, the imbalance between the calculated water lost from the pore fluids and the quantity of hydrous minerals available to incorporate the water must be even greater. This imbalance implies that there is a source of $\mathrm{Cl}$ in the sediments, which could be partly responsible for the observed low $\mathrm{Br} / \mathrm{Cl}$ ratio (Fig. 7A).
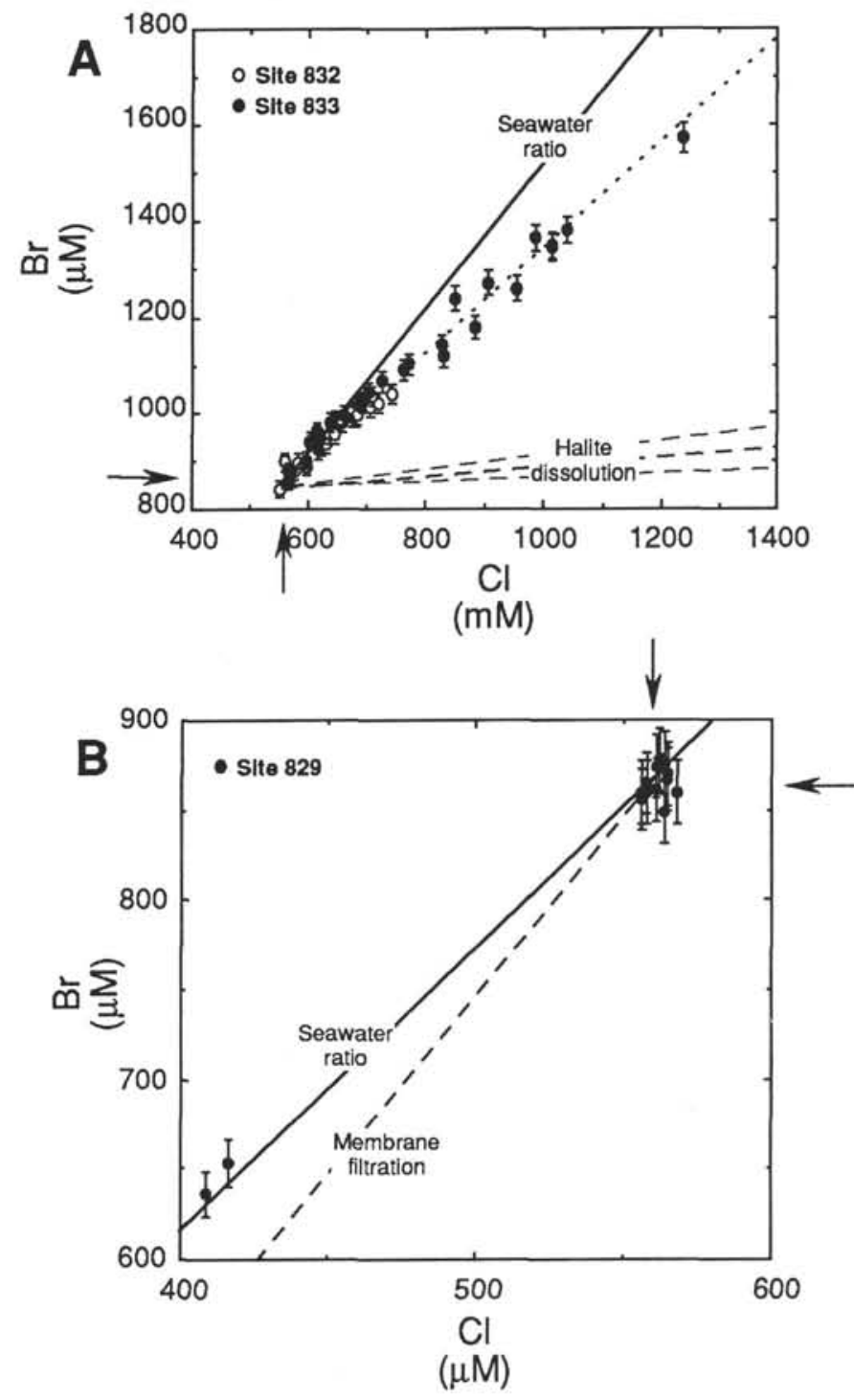

Figure 7. A. Plot of the $\mathrm{Cl}$ vs. Br concentrations from the North Aoba Basin Sites 832 and 833 . The heavy dashed line indicates the $\mathrm{Br} / \mathrm{Cl}$ ratio expected from dissolution of marine halite, and the light dashed lines indicate the possible range in $\mathrm{Br} / \mathrm{Cl}$ ratio from this source. The heavy solid line indicates the $\mathrm{Br} / \mathrm{Cl}$ ratio in seawater. The dotted line indicates the linear regression of all the data $\left(n=52\right.$, slope $\left.=1.05 \times 10^{-3}, r^{2}=0.98\right)$. B. Plot of the $\mathrm{Cl}$ vs. $\mathrm{Br}$ concentrations at accretionary wedge Site 829 . The heavy solid line indicates seawater $\mathrm{Br} / \mathrm{Cl}$ ratio. The dashed line indicates the $\mathrm{Br} / \mathrm{Cl}$ ratio expected from membrane filtration based on results from Kharaka and Berry (1973). The arrows indicate seawater values.

The compositional changes in the major element concentrations (e.g., the exchange of $\mathrm{Ca}$ for $\mathrm{Na}, \mathrm{K}$, and $\mathrm{Mg}$ and the increase in the $\mathrm{Cl}$ concentrations; Fig. 6B) make these pore fluids $\mathrm{CaCl}_{2}$ brines. Similar pore-fluid compositions have previously been reported from both convergent margins and deep ocean basin settings (Egeberg et al., 1990; Lancelot, Larson, et al., 1990; Chambers and Cranston, 1991; Blanc, Hawkins, Parsons, Allan, et al., 1991; Parsons, Hawkins, et al., 1992). These altered pore fluids typically reside in volcanic ash-rich sediment suggesting a mode of formation similar to the North Aoba Basin brines. The North Aoba Basin brines are the most extensively altered and occur in the youngest sediments of all the previously reported brines. At Site 832 the ages of Units II and IV are 
A

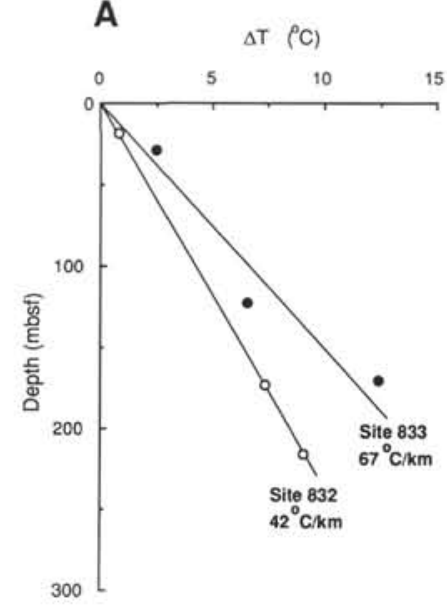

B

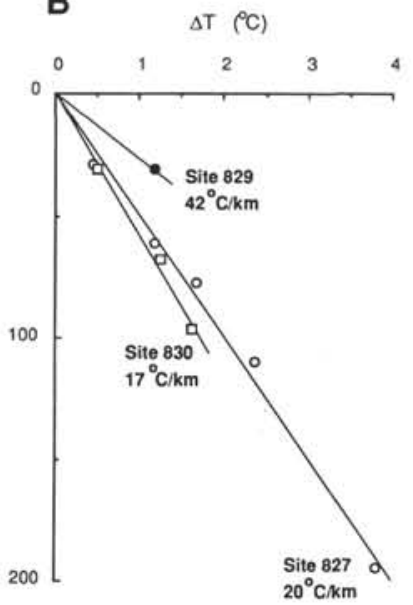

Figure 8. Temperature gradients from North Aoba Basin. (A) Sites 832 and 833 , and (B) accretionary-wedge Sites 827,829 , and 830 . The temperature values were measured using the WSTP tool and are taken from Collot, Greene, Stokking, et al. (1992). $\Delta \mathrm{T}$ is the difference in the measured temperature from the bottom-water temperature.

between 0.45 and $1.45 \mathrm{Ma}$, and at Site 833 the age of Unit III is 1.57 to $1.89 \mathrm{Ma}$ (Staerker, this volume). Assuming that the brines have not formed elsewhere and later flowed into the sediments, the ages of the sediment must be the maximum age of the brine. The young ages indicate that volcanic ash diagenesis and brine formation are very rapid processes.

\section{Diagenetic Controls of Isotopic Compositions}

Because the fractionation factor of oxygen between water and clay and zeolite minerals is $>1$, the alteration of ash to these hydrous minerals will decrease the $\delta^{18} \mathrm{O}$ values of pore fluids in marine sediments (Lawrence et al., 1975; Lawrence and Gieskes, 1981). The decreasing $\delta^{18} \mathrm{O}$ values with depth at Site 833 (Fig. 9A) support the interpretation based on the sediment and pore-fluid compositions of extensive ash alteration in the North Aoba Basin. The linear correlations between $\delta^{18} \mathrm{O}$ values and $\mathrm{Ca}$ and $\mathrm{Cl}$ concentrations also indicate ash alteration, although there are abrupt changes in the slopes of both correlations at 144.9 mbsf (Fig. 10). Although at depths shallower than $144.9 \mathrm{mbsf} \mathrm{Cl}$ concentrations and $\delta^{18} \mathrm{O}$ values correlate well, there is no correlation $\left(r^{2}=0.22\right)$ between $\delta^{18} \mathrm{O}$ values and $\mathrm{Ca}$ concentrations, indicating that different processes control these values in the shallow sediments. The changes in slope are unlikely to be caused by a temperature-dependent decrease in fractionation factor or a more rapid diffusion of oxygen than $\mathrm{Ca}$ or $\mathrm{Cl}$ because both processes would produce smoothly varying plots of $\delta^{18} \mathrm{O}$ vs. Ca and $\mathrm{Cl}$. The change in slope at $144.9 \mathrm{mbsf}$ of the $\mathrm{Ca}-\delta^{18} \mathrm{O}$ plot is greater than the $\mathrm{Cl}-\delta^{18} \mathrm{O}$ plot and the linear correlation between $\mathrm{Ca}$ and $\mathrm{Cl}$ (Figs. 10C and 10D) also changes slope at this depth. The Ca concentrations at depths less than 144.9 mbsf are less than seawater value (Figs. 4 and 5) indicating possible uptake during precipitation of carbonate minerals, but where $\mathrm{Ca}$ concentrations increase rapidly at depths below $144.9 \mathrm{mbsf}$, ash diagenesis appears to be the dominant reaction. This switch from carbonate to ash diagenesis could result from the low alkalinities below $144.9 \mathrm{mbsf}$ (Fig. 5), but could also be caused by a temperature dependent threshold for the conversion of ash into one or more of the authigenic minerals. The $\mathrm{Ca}-\mathrm{Cl}$ correlations in Figure 10D indicate the change in slope occurs between 144.9 and 191.2 mbsf at Site 833 , which suggests a range of 12.7 to $15.8^{\circ} \mathrm{C}$, based on the temperature gradients shown in Figure 8A and assuming a bottom-water temperature of $3^{\circ} \mathrm{C}$. The $\mathrm{Ca}-\mathrm{Cl}$ correlation from Site 832 indicates the same change of slope, at greater depths between

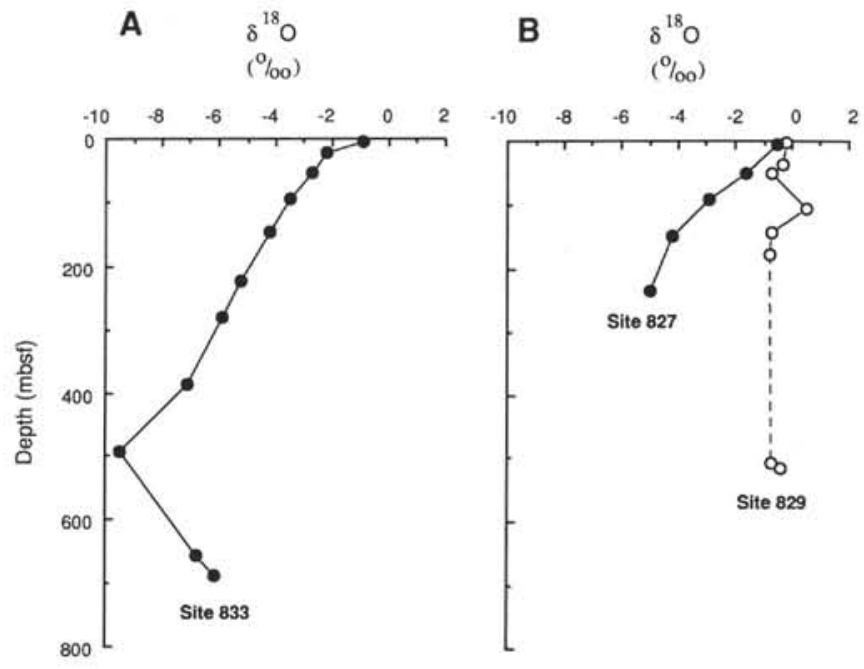

Figure 9. Depth profile of the $\delta^{18} \mathrm{O}$ value of pore water at the North Aoba Basin, (A) Site 833, and (B) accretionary-wedge Sites 827 and 829 .

222.9 and $280.0 \mathrm{mbsf}$, but at similar temperatures of 12.4 to $14.8^{\circ} \mathrm{C}$. There is no lithostratigraphic change at these depths (Fig. 2), and although sedimentation rate decreases from 322 to $148 \mathrm{~m} / \mathrm{m}$.y. at 200 mbsf at Site 833, sedimentation rate is constant from the surface to 350 mbsf at Site 832 (Collot, Greene, Stokking, et al., 1992) suggesting that neither sediment composition or sedimentation rate control these profiles.

Although ${ }^{18} \mathrm{O} /{ }^{16} \mathrm{O}$ ratios of the pore water are extensively altered because of the diagenetic reactions, $\delta \mathrm{D}$ values differ only slightly from values for modern seawater (Fig. 11). Unlike oxygen, the isotopic fractionation of hydrogen between water and clay and zeolite minerals is controlled by a fractionation factor $<1$ (Savin, 1967). The difference in oxygen and hydrogen fractionation factors would increase the $\delta \mathrm{D}$ values and decrease the $\delta^{18} \mathrm{O}$ values of the water, causing the values to plot to the left of the meteoric water line (Fig. 11). At Site 671 and 672, drilled at the Barbados margin, most of the stable isotope ratios also plot to the left of the meteoric water line (Vrolijk et al., 1990), suggesting that volcanic ash diagenetic reactions may be one important control of the stable isotope ratios of the water. At the Barbados forearc, however, the $\delta \mathrm{D}-\delta^{18} \mathrm{O}$ plot exhibits a positive slope that trends along the mean water level, or MWL (Vrolijk et al., 1990), unlike the New Hebrides plot, which exhibits the negative slope expected from volcanic ash diagenesis. The Barbados fluids thus may have a more complex origin than the New Hebrides fluids, including possible mixing with meteoric water.

Both carbonate diagenesis and volcanic ash diagenesis are reflected in the isotopic composition of $\mathrm{Sr}$ in the pore fluids at Site 833 (Fig. 12A). The volcanic ash in the North Aoba Basin has Sr isotope ratios of between 0.703 and 0.704 (Briqueu et al., this volume), thus the ${ }^{87} \mathrm{Sr} /{ }^{86} \mathrm{Sr}$ minima at 95.6 and 494.5 mbsf probably result from isotope exchange with this ash. At 233.5 mbsf the maximum in the ${ }^{87} \mathrm{Sr} /{ }^{86} \mathrm{Sr}$ ratio approaches the value for contemporaneous seawater and corresponds with a carbonate-rich zone containing up to $48 \mathrm{wt} \%$ $\mathrm{CaCO}_{3}$ (Collot, Greene, Stokking, et al., 1992). The ${ }^{87} \mathrm{Sr} /{ }^{86} \mathrm{Sr}$ maximum also corresponds to a maximum in the $\mathrm{Sr}$ concentrations of 2570 $\mu \mathrm{M}$ (Fig. 5), which probably is due to diagenesis of the carbonate. At depths between 600 and 700 mbsf Unit IV also contains $\sim 50 \% \mathrm{CaCO}_{3}$ (Collot, Greene, Stokking, et al., 1992), but the pore-fluid $\mathrm{Sr}$ concentrations and ${ }^{87} \mathrm{Sr} /{ }^{86} \mathrm{Sr}$ ratios remain low, suggesting the older carbonate may have recrystallized, exchanging its $\mathrm{Sr}$ isotopes.

Mixing between two end-member fluids with different $\mathrm{Sr}$ concentrations and isotope ratios results in an exponential mixing curve (see Faure, 1986, for derivation) that forms a straight line when the isotope 

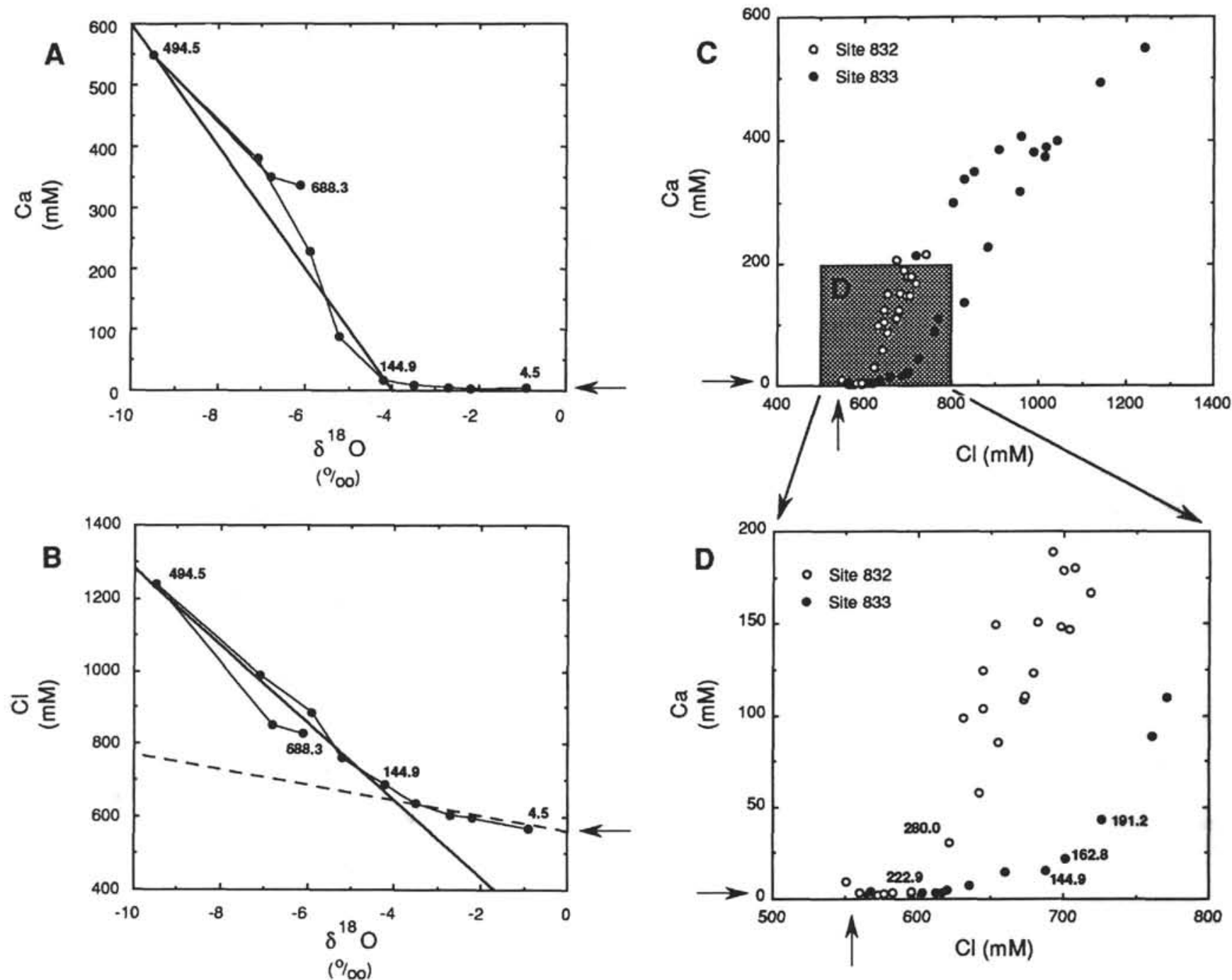

Figure 10. Plot of the $\delta^{18} \mathrm{O}$ value of the pore water vs. (A) Ca concentration, and (B) Cl concentration for North Aoba Basin Site 833. The dashed line indicates the linear regression of the $\delta^{18} \mathrm{O}-\mathrm{Cl}$ data from the shallow pore fluids $\left(n=4\right.$, slope $\left.=-26, r^{2}=0.99\right)$, the solid lines indicate the linear regressions of the $\delta^{18} \mathrm{O}-\mathrm{Cl}$ and $\delta^{18} \mathrm{O}$-Ca data from the deep pore fluids ( $n=7$, for A.: slope $=-395, r^{-2}=0.94$; for B.: slope $\left.=-104, r^{2}=0.94\right)$. C, D. Plots of Cl vs. Ca concentrations for North Aoba Basin Sites 832 and 833. All data are plotted in (C), while a selected range of data is plotted in (D) in order to emphasize the depth of the change in slope. The numbers beside the data in A, B, and D indicate the depth (mbsf) of selected samples. Arrows indicate seawater values.

ratios are plotted versus $1 / \mathrm{Sr}$ concentrations. At depths shallower than 144.9 mbsf such a plot is not linear (Fig. 13), indicating that the $\mathrm{Sr}$ concentrations and isotope ratios result from mixing of more than two end-members. Above 144.9 mbsf the pore-fluid Sr likely would be influenced by three sources: volcanic ash, carbonates and seawater. Below 144.9 mbsf the data plots a straight line, indicating the presence of only two end members, probably pore fluids altered by ash or carbonate diagenesis. The end member with high $\mathrm{Sr}$ concentration and radiogenic isotope ratio extrapolates to a value equal to that for the carbonate in the North Aoba Basin sediment. The other end member appears to have both low $\mathrm{Sr}$-isotope ratios and low $\mathrm{Sr}$ concentrations. Assuming that the low $\mathrm{Sr}$-isotope ratios result from ash diagenesis, pore fluid with completely exchanged isotopes (i.e., values between 0.703 and 0.704 ) would have $\mathrm{Sr}$ concentrations ranging between 60 and $80 \mu \mathrm{M}$ (Fig. 13). These concentrations, lower than seawater value, indicate that $\mathrm{Sr}$ is lost from the pore fluids during ash diagenesis and the generation of authigenic minerals, for example in the shallow pore fluids where $\mathrm{Sr}$ concentrations are less than seawater value (Table 1). Assuming that one of the authigenic clay minerals is smectite, a possible site for this $\mathrm{Sr}$ could be substitution for $\mathrm{Ca}$ in the smectite.

\section{Accretionary Wedge (Sites 827, 829, 830)}

\section{Diagenetic Controls of Solute Concentrations and Isotopic Compositions}

At depths less than $100 \mathrm{mbsf}$ at all three accretionary wedge sites the pore fluid composition reflects organic matter diagenesis; $\mathrm{SO}_{4}$ concentration decreases sharply, while $\mathrm{NH}_{4}, \mathrm{PO}_{4}$, and alkalinity concentrations increase (Figs. 14, 15, 16). At all three sites, however, the $\mathrm{SO}_{4}$ concentration increases below the depth of the $\mathrm{SO}_{4}$ minimum. Because of the extreme care that was taken during handling of the samples the increase in $\mathrm{SO}_{4}$ concentration is unlikely a result of contamination by the drilling fluid. The deep $\mathrm{SO}_{4}$-rich pore fluids also contain low $\mathrm{Mg}$ concentrations, further indicating there has been little contamination. (Figs. 14, 15, 16). At Sites 827 and 830 the deep $\mathrm{SO}_{4}$-rich pore fluids were recovered from volcanic breccia units that contain little organic carbon (Fig. 2). Similar to the deep $\mathrm{SO}_{4}$-rich pore fluids in the North Aoba Basin, this observed increase in $\mathrm{SO}_{4}$ concentration probably reflects seawater $\mathrm{SO}_{4}$ trapped during the burial of the organic carbon-poor volcanogenic sediment. 


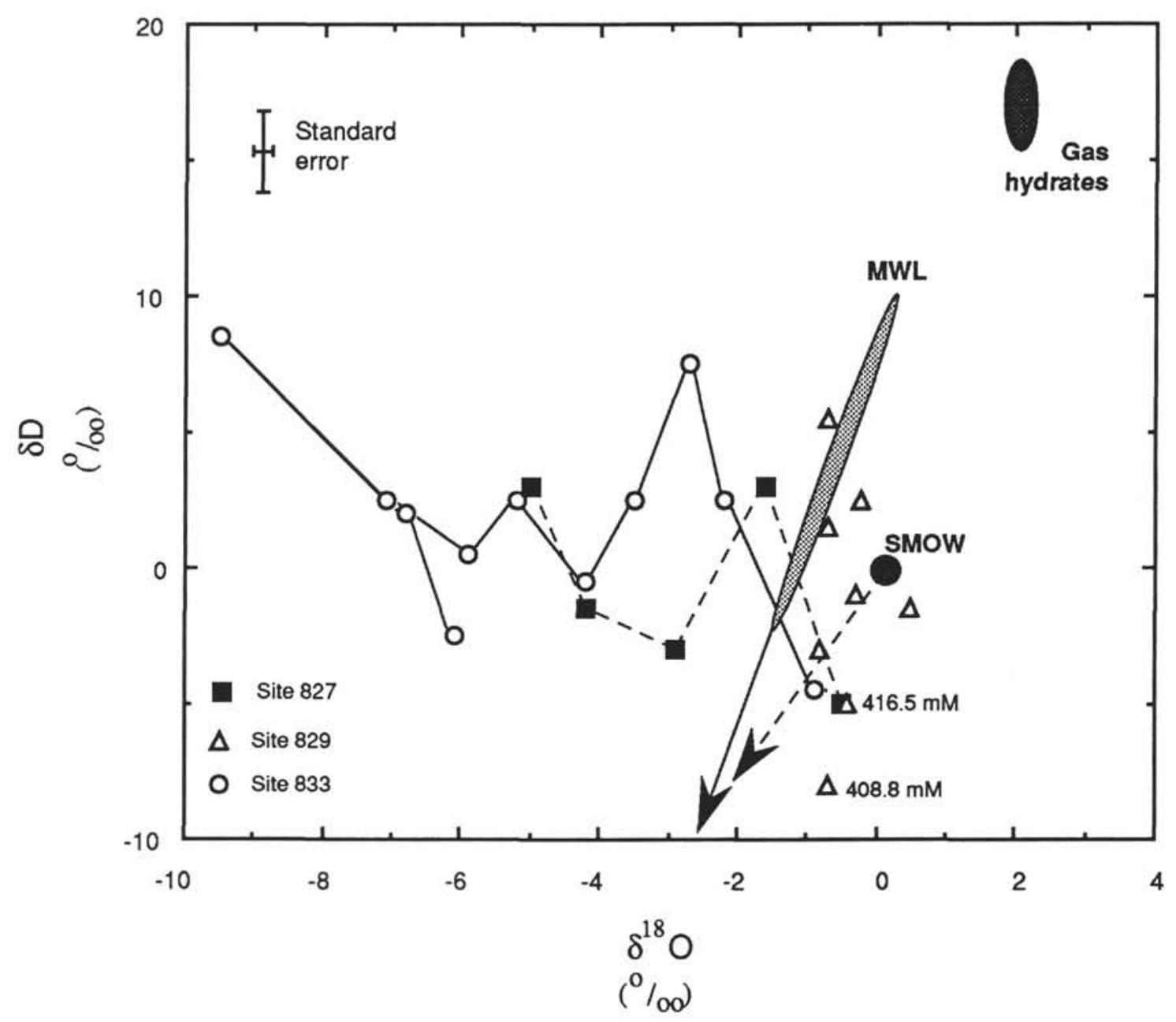

Figure 11. Plot of the pore water $\delta^{18} \mathrm{O}$ vs. $\delta \mathrm{D}$ ratios for the North Aoba Basin Site 833 and the accretionary wedge Sites 827 and 829 . The solid line with an arrow indicates the meteoric water line (MWL; Craig, 1961), and the dashed line with the arrow indicates the $\delta \mathrm{D}-\delta^{18} \mathrm{O}$ correlation $\left(r^{2}=0.95\right)$ for meteoric water from fifteen tropical islands (Dansgaard, 1964). The lightly shaded field on the MWL indicates the expected $\delta \mathrm{D}$ and $\delta^{18} \mathrm{O}$ values for low-latitude meteoric water (Dansgaard, 1964). The two low-Cl samples from Site 829 are labeled with their concentrations.

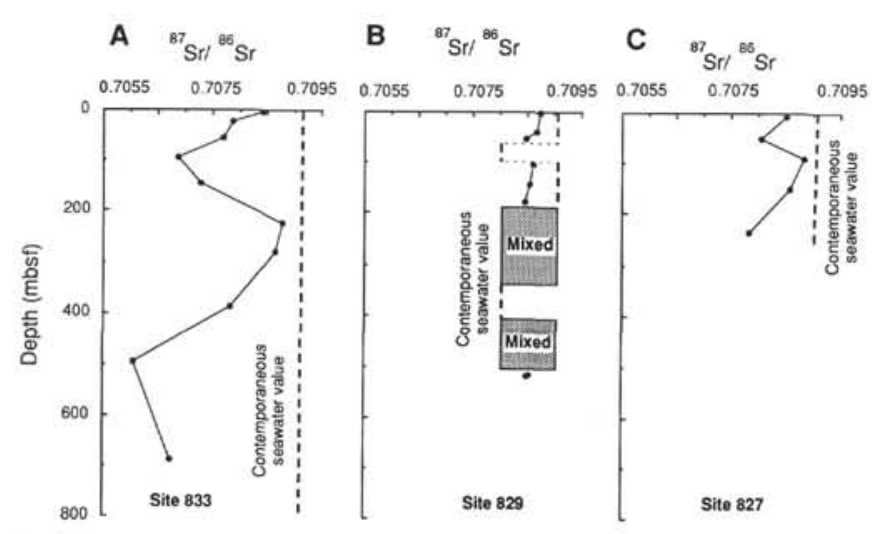

Figure 12. Depth profile of the ${ }^{87} \mathrm{Sr} /{ }^{86} \mathrm{Sr}$ isotope ratios of the dissolved $\mathrm{Sr}$ for (A) Site 833, (B) Site 829, and (C) Site 827. The dashed lines indicate the contemporaneous seawater ${ }^{87} \mathrm{Sr} /{ }^{86} \mathrm{Sr}$-isotope ratios, and at depths at Site 829 where sediment ages range from Oligocene to Pleistocene due to tectonic mixing the contemporaneous seawater ${ }^{87} \mathrm{Sr} /{ }^{86} \mathrm{Sr}$ ratios (Elderfield 1986; Hodell et al., 1991) are shown by shaded boxes.
In the accretionary-wedge pore fluid, $\mathrm{Cl}$ concentrations are characterized by values both greater and less than seawater value (Fig. 17). At Sites 827 and 830 the concentrations reach maxima of 628 $\mathrm{mM}$ and $620 \mathrm{mM}$, which are intermediate between seawater value and the maxima reached in the North Aoba Basin pore fluids. At Site 829 above 200 mbsf the $\mathrm{Cl}$ concentration increases only slightly above seawater value, reaching a maximum concentration of $568 \mathrm{mM}$. Below 400 mbsf the concentration decreases to a minimum of 409 $\mathrm{mM}$. These are the only pore fluids sampled from the New Hebrides margin with $\mathrm{Cl}$ concentrations less than seawater value, an attribute common to convergent margins (Kastner et al., 1991).

At Sites 827 and 830 the major element concentrations show the same exchange of $\mathrm{Ca}$ for $\mathrm{Na}, \mathrm{K}$, and $\mathrm{Mg}$ as the North Aoba Basin pore fluids (Fig. 18). This exchange is best explained by the alteration of volcanic ash to hydrous minerals which could also cause the increased $\mathrm{Cl}$ concentrations at these two sites (Fig. 17). At Site 827 the $\delta^{18} \mathrm{O}$ values and ${ }^{87} \mathrm{Sr} /{ }^{86} \mathrm{Sr}$ ratios also indicate large amounts of ash diagenesis (Figs. 9B and $12 \mathrm{C}$ ). The $\delta^{18} \mathrm{O}$ values decrease with depth with approximately the same gradient as at Site 833 (Fig. 9). Although the $\delta \mathrm{D}$ values are scattered at Site 827 , most of these pore fluids plot to the left of the meteoric water line, similar to Site 833 (Fig. 11). The ${ }^{87} \mathrm{Sr} /{ }^{86} \mathrm{Sr}$ ratios show a wide range of values (Table 3 ), but they are all 


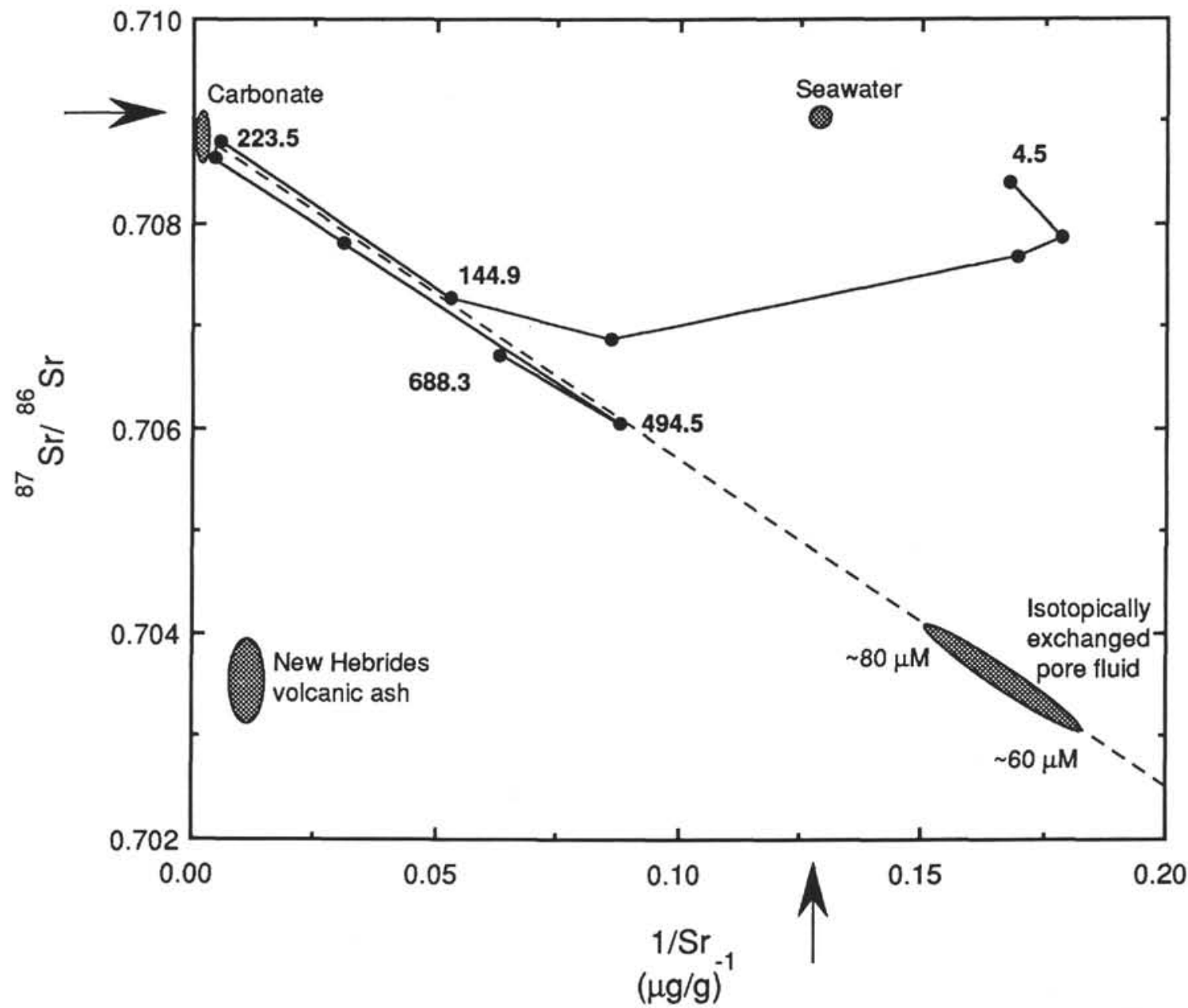

Figure 13. Plot of the ${ }^{87} \mathrm{Sr} /{ }^{86} \mathrm{Sr}$ isotope ratio vs. $1 / \mathrm{Sr}$ concentration of dissolved $\mathrm{Sr}$ for the North Aoba Basin Site 833 . The $1 / \mathrm{Sr}$ concentrations were converted from units of $\mu \mathrm{M}^{-1}$ to $(\mu \mathrm{g} / \mathrm{g})^{-1}$, assuming a constant pore-fluid density of $1023 \mathrm{~kg} / \mathrm{m}^{3}$. The isotope ratios and $1 / \mathrm{Sr}$ concentrations of possible end-members are shown on the plot, including modern seawater, volcanic ash in the New Hebrides sediments, carbonate minerals, and pore fluid with isotope ratios identical to the New Hebrides ash. The dashed line indicates the linear regression of data from pore fluid at $144.9 \mathrm{mbsf}$ and deeper $\left(n=6\right.$, slope $\left.=-0.03215, r^{2}=0.99\right)$. Based on this regression line, pore fluid with ${ }^{87} \mathrm{Sr} /{ }^{86} \mathrm{Sr}$ isotope ratios between 0.704 and 0.703 should have $\mathrm{Sr}$ concentrations between 80 and $60 \mu \mathrm{M}$. The bold numbers indicate the depths (mbsf) of the samples. The arrows indicate modern seawater value.

lower than the contemporaneous seawater value (Fig. 12C), suggesting isotopic exchange with the ash. The Sr concentrations are somewhat elevated above seawater values (Table 1). If the ash acts as a sink for $\mathrm{Sr}$, as suggested by Figure 13, the elevated $\mathrm{Sr}$ concentrations indicate that some $\mathrm{Sr}$ was released during carbonate diagenesis, thereby preventing an even greater decrease in the isotope ratios.

\section{Evidence for Fluid Flow}

At depths less than $200 \mathrm{mbsf}$ at Site 829 the major element concentrations are less altered from seawater values than at Sites 827 or 830 (Fig. 18), although Sites 827 and 829 are separated by only 1.5 $\mathrm{km}$. Because the geothermal gradient at Site 829 is twice the gradient at Sites 827 and 830 (Fig. 6B), these compositional differences do not result from elevated temperatures increasing the reaction rates at Sites 827 and 830 . At these depths the sediments at Site 829 are tectonically mixed by thrusting and consist of Pleistocene ash-rich units and Oligocene to Pleistocene ash-rich carbonate units (Fig. 2). The thrust faults are located at the depths of changes in some of the pore-fluid gradients. The most strongly altered profiles are the $\mathrm{Ca}, \mathrm{Sr}, \mathrm{Li}, \mathrm{SO}_{4}$. and alkalinity concentrations and the $\mathrm{Ca} / \mathrm{Mg}$ ratio (Fig. 19), although the $\mathrm{NH}_{4}$ and $\mathrm{PO}_{4}$ gradients also exhibit inflections at the same depths (Fig. 15). Although the sharply increasing $\mathrm{Ca} / \mathrm{Mg}$ ratios could be caused by ash diagenesis in Units I and III, the $\delta^{18} \mathrm{O}$ values and $\mathrm{Sr}$ isotope ratios indicate only minor ash diagenesis (Figs. 9B and 12B); unlike Site 827 , these isotope ratios change only slightly from modern seawater values. The radiogenic ${ }^{87} \mathrm{Sr} /{ }^{86} \mathrm{Sr}$ ratios and the elevated $\mathrm{Sr}$ concentrations indicate that much of the $\mathrm{Sr}$ is derived from diagenesis of Tertiary carbonate which also could control the $\mathrm{Ca}$ and $\mathrm{Ca} / \mathrm{Mg}$ profiles (Fig. 19). Because Units I and III are carbonate-poor (Fig. 2), the carbonate diagenesis probably occurs within carbonate-rich Units II and IV through VI.

The higher carbonate content of the Site 829 sediment could be responsible for its different pore-fluid composition from Sites 827 and 830 , but these differences could also reflect fluid flow at Site 829 with less, or no, flow at Sites 827 and 830 . The small changes in $\delta^{18} \mathrm{O}$ values, ${ }^{87} \mathrm{Sr} /{ }^{86} \mathrm{Sr}$ ratios, and $\mathrm{Cl}$ concentrations, even within the ashrich Units I and III at Site 829 , indicate there is less diagenetic alteration to the pore-fluid composition than at Site 827. The altered pore-fluid chemistry at Site 827 could reflect a long residence time and thus little flow and a low water/rock ratio in those sediments. Other indications of more flow at Site 829 than at 827 include porosity 

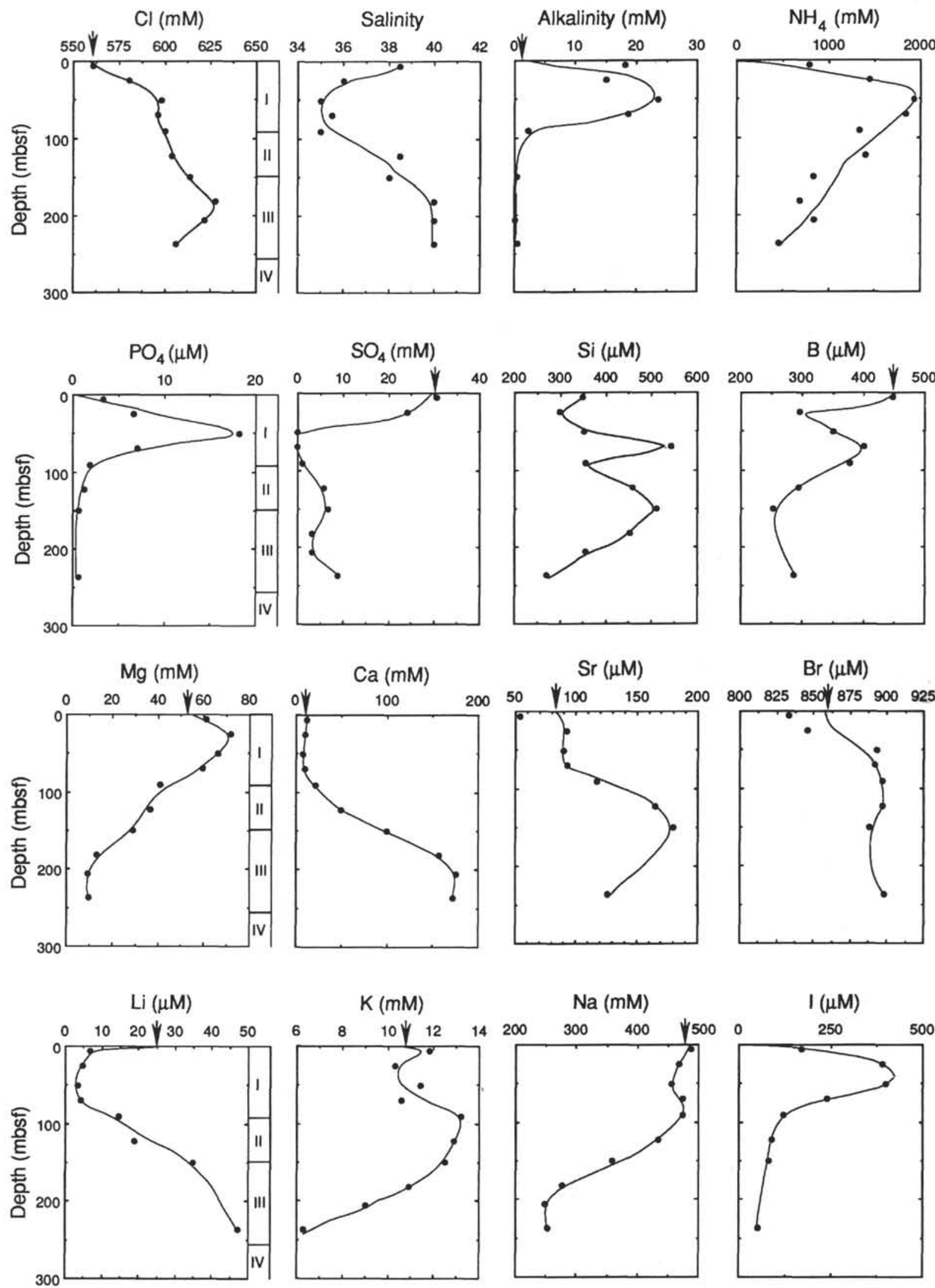

Figure 14. Depth profiles of pore fluid species and unit thicknesses for accretionary wedge Site 827. Arrows indicate seawater composition. 

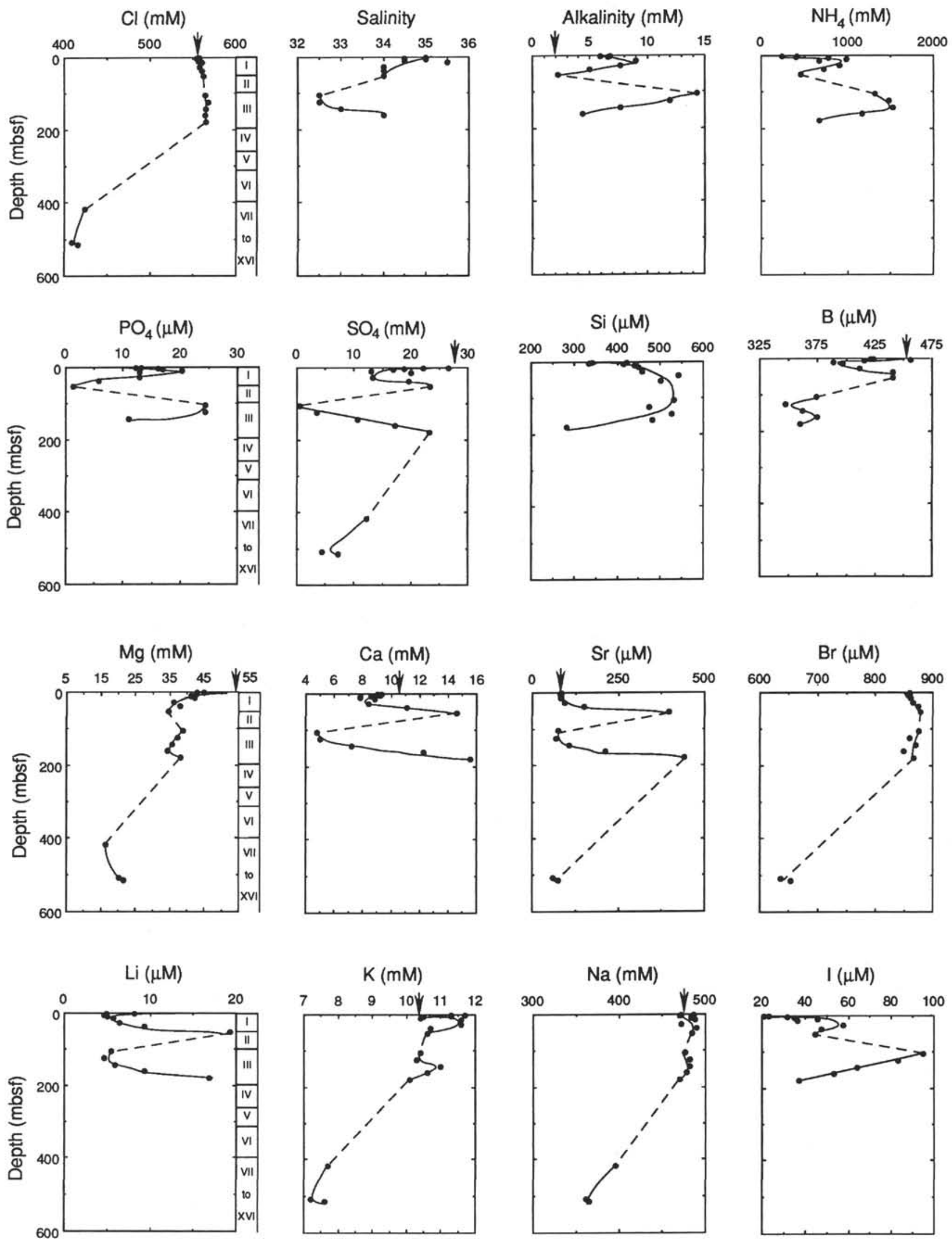

Figure 15. Depth profiles of pore fluid species and unit thicknesses for accretionary wedge Site 829. Because Units VII to XVI are thin (Fig. 2) their boundaries are not marked. Arrows indicate seawater composition. 

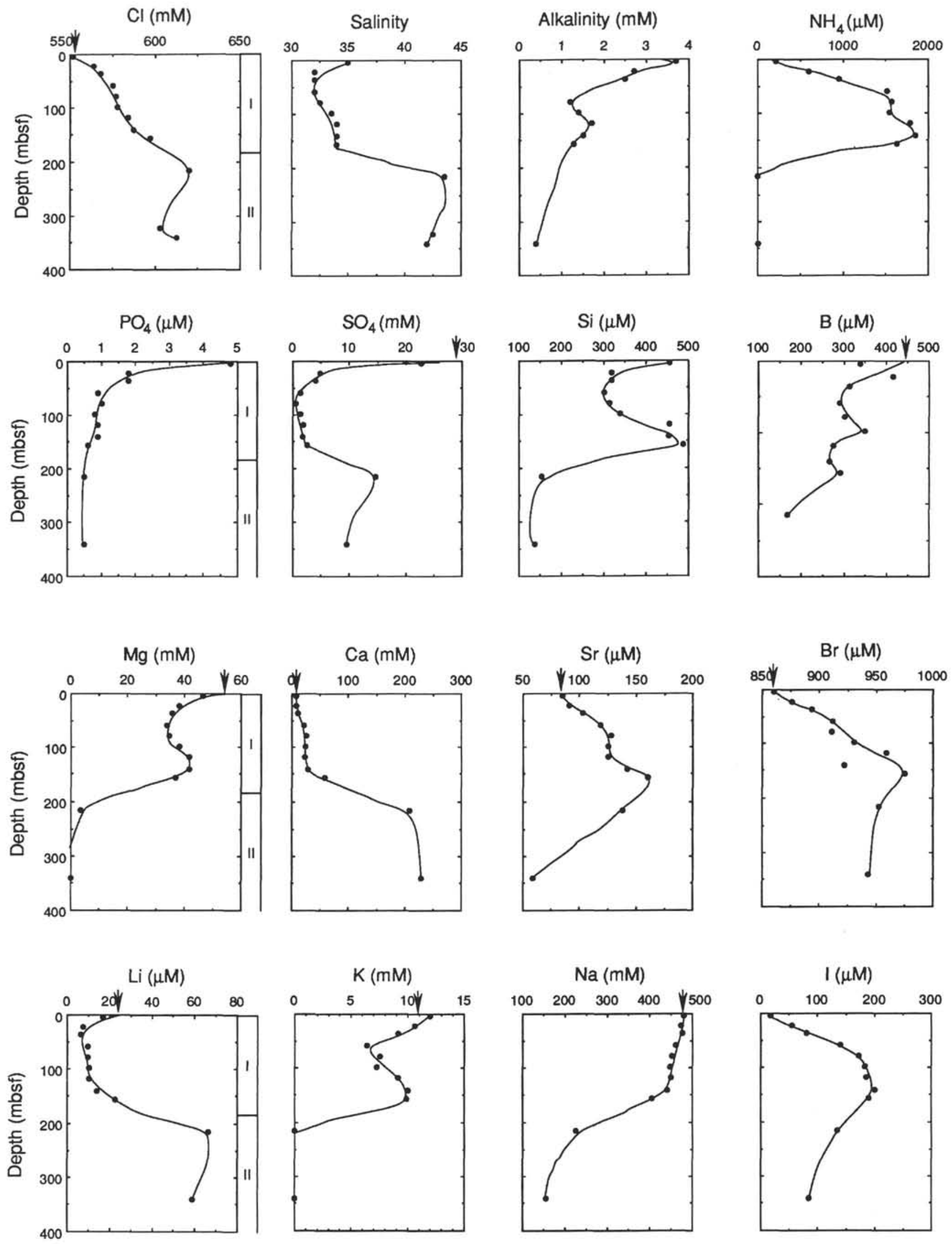

Figure 16. Depth profiles of pore fluid species and unit thicknesses for accretionary wedge Site 830. Arrows indicate seawater composition. 


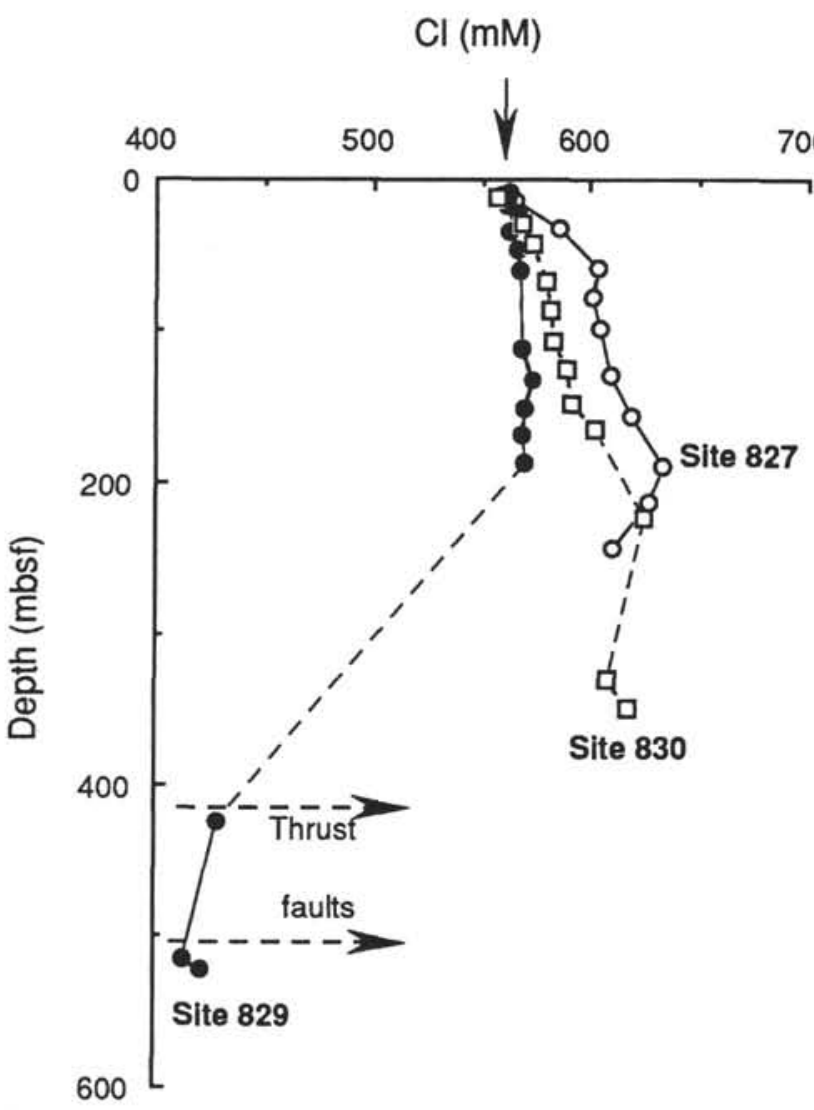

Figure 17. Depth profile of $\mathrm{Cl}$ concentrations in the three accretionary wedge Sites 827,829 , and 830 . The short horizontal dashed lines with arrows indicate the location of thrust faults at Site 827 (Fig. 2). The arrow indicates seawater value.

and temperature distributions. Modelling indicates that porosity reduction, and thus fluid flow, in accretionary wedges increases from the trench axis toward the volcanic arc (Bekins and Dreiss, 1992). Although Site 829 is closer to the trench than Site 827 , porosity reduction is more rapid even within the ash-rich units. The higher temperature gradient at Site 829 than at Sites 827 or 830 could be caused by upward flow of warm water. Likely conduits for flow at Site 829 would be the major thrust faults in the upper $200 \mathrm{~m}$ (Fig. 2).

At depths between 200 and 400 mbsf at Site 829 , the mixed sediments of Units IV through VI have low porosity $(\sim 30 \%)$ and water content of approximately 15 to $20 \%$ (Leonard and Ask, this volume). The pore-fluid profiles shown in Figure 15 thus lack data between 200 and $400 \mathrm{mbsf}$. Of the five pristine samples collected and squeezed at depths below 379 mbsf only three yielded fluid (Collot, Greene, Stokking, et al., 1992). These three samples are located less than 20 $\mathrm{m}$ below major thrust faults, while the samples yielding no fluid are located less than $25 \mathrm{~m}$ above thrust faults. This apparent structural control of the fluid distribution is supported by water content measurements showing two to three times the background water contents in the vicinity of faults. Although some of these elevated water content measurements could be caused by infiltration of drilling fluid, the pore-fluid compositions, discussed in detail below, indicate that some of the fluid is uncontaminated formation fluid.

The deep fluids at Site 829 are characterized by $\mathrm{Cl}$ concentrations less than seawater value (Fig. 17), an attribute exhibited by all convergent margins studied (Kastner et al., 1991). Results from a variety of convergent margins (e.g., Blanc et al., 1988, 1991; Gieskes et al., 1990; Kastner et al., 1990; Le Pichon et al., 1990; Gieskes et al., 1993; Mottl et al., 1993) suggest that the low-Cl fluids could originate from several sources. These sources include dilution by a pure water phase released

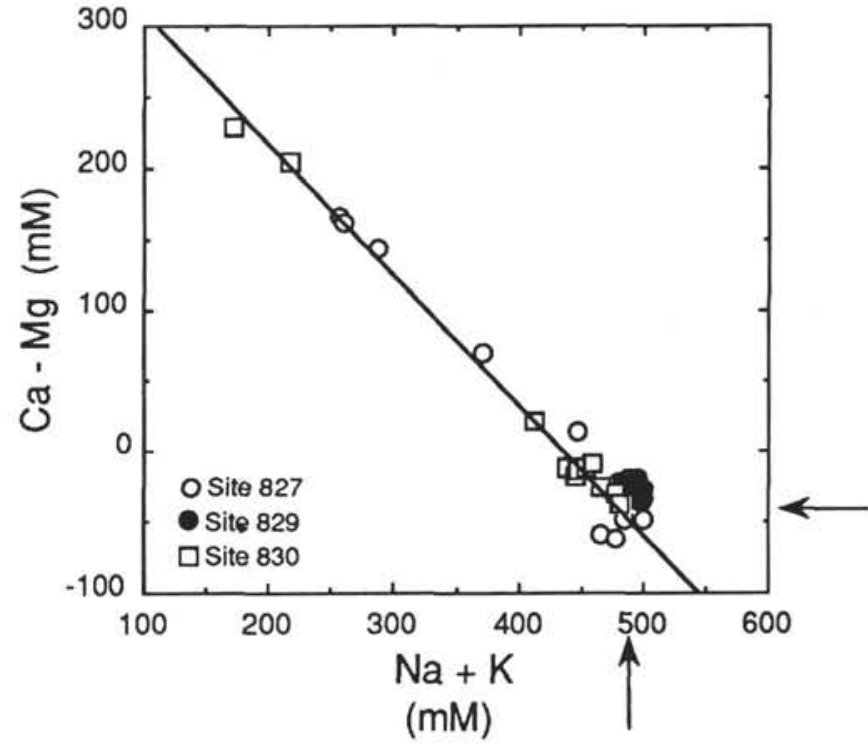

Figure 18. Plot of the $\mathrm{Ca}$ minus $\mathrm{Mg}$ concentration vs. the $\mathrm{Na}$ plus $\mathrm{K}$ concentration for the accretionary wedge sites. The arrows indicate seawater values. The solid line indicates a slope of -1
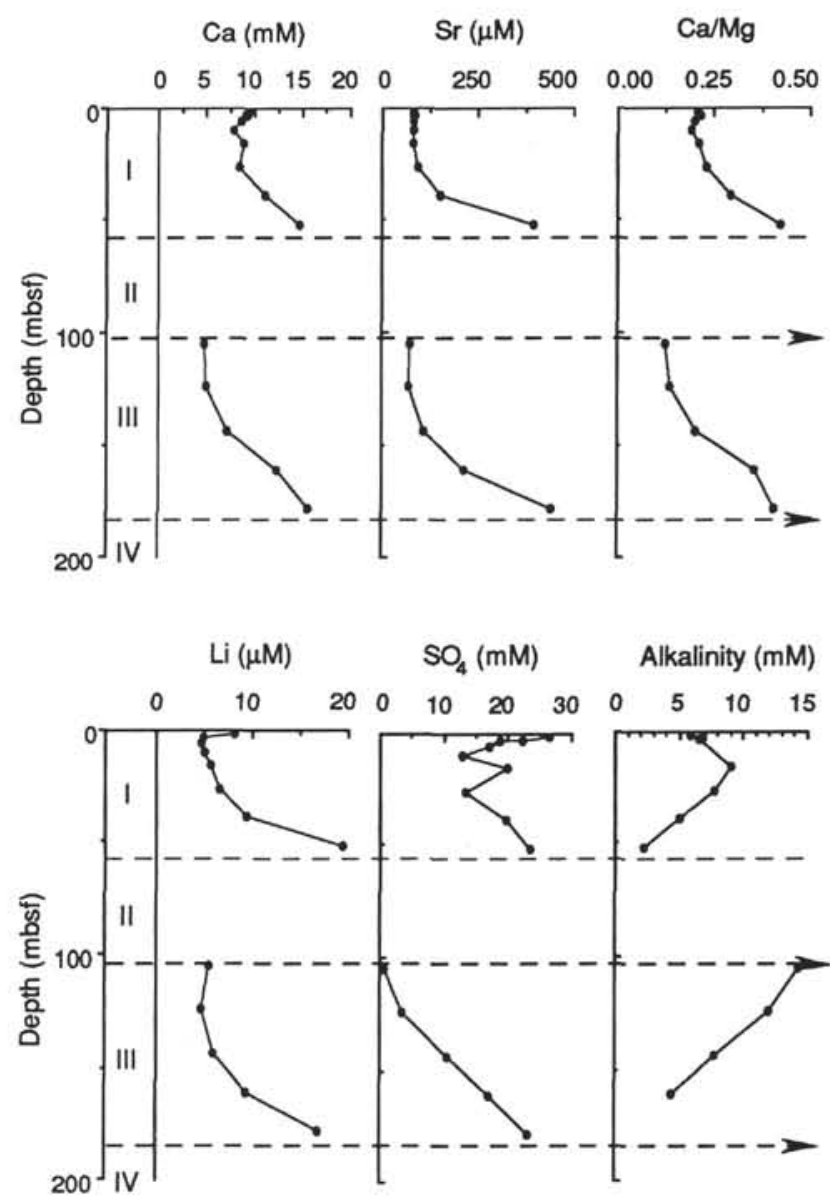

Figure 19. Depth profiles of the $\mathrm{Ca}, \mathrm{Sr}, \mathrm{Li}, \mathrm{SO}_{4}$, and alkalinity concentrations and the $\mathrm{Ca} / \mathrm{Mg}$ ratio in the sediments above $200 \mathrm{mbsf}$ at the accretionary wedge Site 829 . The boundaries between Units I, II, III, and IV are shown by dashed lines; lines with arrows indicate thrust faults. 


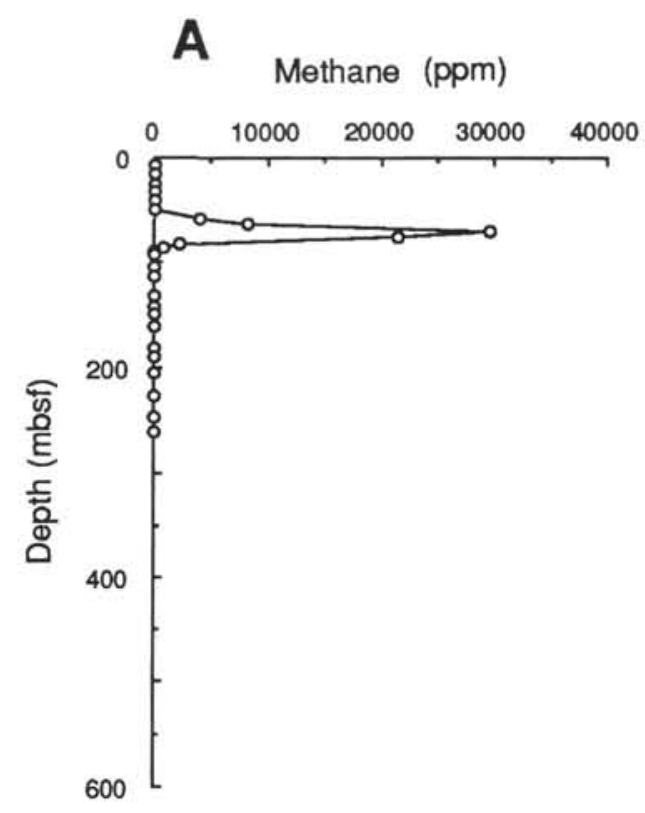

Site 827

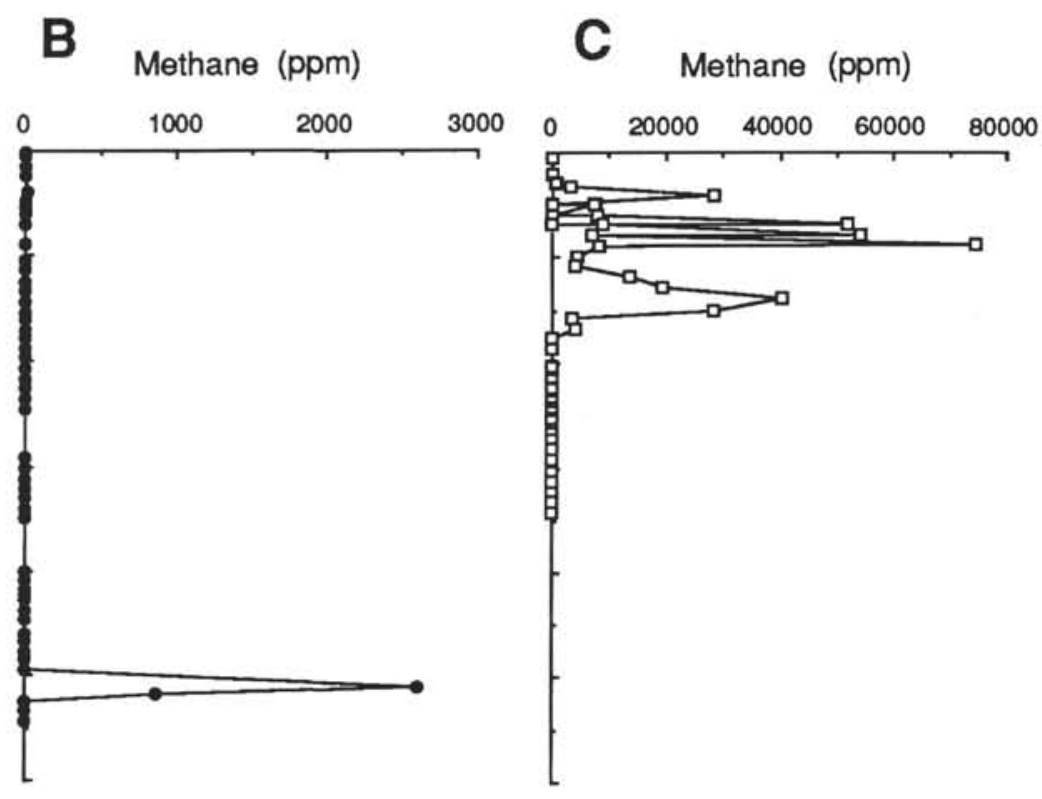

Site 829
Site 830

Figure 20. Depth profile of methane concentrations at the accretionary wedge Sites (A) 827, (B) 829, and (C) 830 . The units of ppm are on a volume basis $(\mu \mathrm{l} / \mathrm{L})$ and reflect liberation of methane at $70^{\circ} \mathrm{C}$ from $5 \mathrm{~cm}^{-3}$ of sediment into a $21 \mathrm{~cm}^{-3}$ vial (Collot, Greene, Stokking, et al., 1992).

during the dissolution of gas hydrates or dehydration of hydrous minerals such as clays and zeolites, dilution by mixing with meteoric water, and also possibly membrane ion filtration (for a description of this process see Kharaka and Berry, 1973; Graf, 1982).

Experimental work shows that during high-pressure filtration, $\mathrm{Cl}$ passes more easily through clay membranes than $\mathrm{Br}$, thereby decreasing the $\mathrm{Br} / \mathrm{Cl}$ ratio in the low- $\mathrm{Cl}$ fluid by up to a factor of $10 \%$ (Kharaka and Berry, 1973). In the shallow pore fluids at Site 829, the $\mathrm{Cl}$ and $\mathrm{Br}$ concentrations are near seawater value and thus the $\mathrm{Br} / \mathrm{Cl}$ ratio is nearly constant at approximately seawater value. The low $\mathrm{Cl}$ fluid also has a $\mathrm{Br} / \mathrm{Cl}$ ratio identical, within error, to seawater ratio, suggesting that membrane filtration is not the cause of the low $\mathrm{Cl}$ concentrations (Fig. 8B). The seawater $\mathrm{Br} / \mathrm{Cl}$ ratio implies that the low $\mathrm{Cl}$ concentration results from simple dilution of pore fluids that originally were characterized by seawater $\mathrm{Br} / \mathrm{Cl}$ ratio.

The dilution is unlikely to have been caused principally by gas hydrate dissociation, although the estimated formation temperatures of about $20^{\circ} \mathrm{C}$ at the depth of the low $\mathrm{Cl}$ fluids are within the stability field of gas hydrates. Assuming a stoichiometry for gas hydrates of $\mathrm{CH}_{4} \cdot 5.75 \mathrm{H}_{2} \mathrm{O}$ (Davidson et al., 1978), approximately 2.4 moles of methane per liter of pore water would be liberated in order to produce a sufficient quantity of water to cause the observed $25 \%$ decrease in $\mathrm{Cl}$ concentrations. For sediments with $20 \%$ porosity and assuming that no methane is lost during the core handling and that all the methane is liberated during the head-space procedure, the observed maximum of $2500 \mathrm{ppm}$ methane (Fig. 20B) converts to a concentration of $\sim 0.3 \mathrm{mM}$ methane. Because of the difficulty associated with collecting pristine gas samples, this concentration estimate is probably good only to within an order of magnitude, but it is nearly 4 orders of magnitude less than the amount of methane that would be released from enough gas hydrate to account for the observed dilution. Dense gas hydrate layers appear to be missing from the region because bottom simulating reflectors have not been observed from seismic investigations (Fisher et al., 1991). Gas hydrates are characterized by positive $\delta^{18} \mathrm{O}$ and $\delta \mathrm{D}$ values, for example, water from a dissociated gas hydrate collected from the Peru margin had $\delta^{18} \mathrm{O}$ and $\delta \mathrm{D}$ values of $+1.8 \%$ and $+17.8 \%$, respectively (Kastner et al., 1990), and $\delta^{18} \mathrm{O}$ values up to $+2.6 \%$ of pore water at the Guatemala margin are attributed to gas hydrate dissociation (Hesse and Harrison, 1981). In contrast to these positive values, the low- $\mathrm{Cl}$ fluid at Site 829 is characterized by slightly negative $\delta^{18} \mathrm{O}$ and $\delta \mathrm{D}$ values (Fig. 11). The low methane concentrations and $\delta \mathrm{D}$ and $\delta^{18} \mathrm{O}$ values thus indicate that the dilution does not result only from gas hydrate dissociation.

In order for meteoric water to dilute the $\mathrm{Cl}$ concentrations, there must be a source for the water. For example, mixing with meteoric water possibly occurs at the Peru margin (Kastner et al., 1989) and the Barbados accretionary wedge (Le Pichon et al., 1990, 1991), both of which are close to the South American continent. The New Hebrides Island Arc, where only a small area of land is exposed above sea level, is $2000 \mathrm{~km}$ from the nearest continent, Australia (Fig. 1), and the island nearest to Site 829, Espiritu Santo, emerged above sea level only 500,000 years ago in conjunction with the collision of the DEZ (Taylor, 1992). A direct conduit from Espiritu Santo Island to Site 829 , however, could be provided by several faults that trend perpendicular to the trench axis and which may be connected to onshore faults (Collot and Fisher, 1991). In order for meteoric water to have flowed from Espiritu Santo Island to Site 829, a distance of $\sim 50 \mathrm{~km}$, flow rates on the order of $90 \mathrm{~cm} / \mathrm{yr}$ would be required.

An additional possible source of low $\mathrm{Cl}$ fluids is from the dehydration of clay and zeolite minerals. The observed $25 \%$ decrease in $\mathrm{Cl}$ concentration from seawater value (Fig. 17) requires approximately $0.05 \mathrm{~m}^{3}$ water $/ \mathrm{m}^{3}$ sediment for sediment with $\sim 20 \%$ porosity such as at Site 829. Kastner et al. (1991) calculated that complete dehydration of sediment containing $20 \mathrm{wt} \%$ smectite and $30 \%$ porosity would provide $0.021 \mathrm{~m}^{3}$ water $/ \mathrm{m}^{3}$ sediment. Higher clay contents, breakdown of clay minerals and the release of structural water and dehydration of the oceanic crust would provide additional water. Sufficient water for the observed dilution at Site 829 thus could be produced through mineral dehydration alone.

The $\delta^{18} \mathrm{O}$ and $\delta \mathrm{D}$ values indicate several sources may contribute to the low-Cl fluid. The $\delta^{18} \mathrm{O}$ value of water released from hydrous minerals should be positive, but the observed $\delta^{18} \mathrm{O}$ values of the low- $\mathrm{Cl}$ fluids are negative, with values of $-0.7 \%$ and $-0.4 \%$ (Table 2 ). These values are heavier relative to their $\delta \mathrm{D}$ values than those 
expected from meteoric water at low latitudes and on tropical islands (Fig. 11). The isotope ratios therefore suggest that the source of the fluid is a mixture of meteoric water and water released during mineral dehydration. Because precise isotopic values of the possible mineral and meteoric water sources are unknown, the relative proportions from each source cannot be evaluated.

Based on the temperature gradient shown in Figure 6B, temperatures of $\sim 20^{\circ} \mathrm{C}$ at 400 to $500 \mathrm{mbsf}$ are $\sim 40^{\circ} \mathrm{C}$ lower than those required for dehydration of interstitial or channel water (Colton-Bradley, 1987). Such temperatures will occur at $>1.5 \mathrm{~km}$ burial depth at Site 829 and even greater depths for Site 827 and 830 because of their lower temperature gradients. Advection of the deep fluid is required in order for water released below $1.5 \mathrm{~km}$ to dilute the fluids at $400 \mathrm{mbsf}$; elevated water contents near fault zones suggest that advection occurs along the fault planes. Advection along faults of low- $\mathrm{Cl}$ water has previously been proposed to explain low- $\mathrm{Cl}$ and methane-rich fluid found in the Barbados accretionary wedge (Blanc et al., 1988, 1991; Gieskes et al., 1990; Vrolijk et al., 1990, 1991). The methane in the Barbados water has been identified as thermogenic based on its isotope ratio and the presence of heavier hydrocarbons supporting a deep origin of the fluids. In the New Hebrides margin, the low-Cl fluid also contains methane (Fig. 20). No heavier hydrocarbons were observed and the isotope ratios of this gas have not been measured, and thus the gas origin, whether biogenic or thermogenic, is unknown.

\section{CONCLUSIONS}

Results from the New Hebrides margin show that fluid with seawater composition can be extensively and rapidly altered into a $\mathrm{CaCl}_{2}$ brine simply through exchange reactions with the solid phases and authigenesis of hydrous minerals. Assuming that the North Aoba Basin brines are formed in situ and have not flowed from elsewhere, at a maximum they should be of the same age as the surrounding sediments. These maximum ages are only between 0.45 and $1.89 \mathrm{Ma}$ at the burial depths of volcanic breccias at Sites 832 and 833. Minor element concentrations are also altered by this diagenesis. For example, $\mathrm{Sr}$ appears to be incorporated into authigenic silicate minerals. In contrast with $\mathrm{Sr}$, however, $\mathrm{Li}$ appears to be only initially incorporated into the solid phases; subsequently, at greater burial depths it seems to be released. This change in behavior could be due to temperaturedependent reactions, as observed experimentally by Seyfried et al. (1984), which shows that $\mathrm{Li}$ is removed from pore fluids and incorporated into solids at temperatures $<150^{\circ} \mathrm{C}$. Results from the New Hebrides margin suggest that $\mathrm{Li}$ may be leached from solid phases at temperatures less than experiments indicate: $\mathrm{Li}$ maxima occur at depths of only $300 \mathrm{mbsf}$ where temperatures are probably $<35^{\circ} \mathrm{C}$.

The New Hebrides margin also provides additional information about the hydrologic behavior of fluids at convergent margins. Fluid flow and the lack of flow appear to affect the extent of chemical and isotopic alteration of the accretionary wedge pore fluid. Fluid flow seems to be fault controlled, and those regions with few or no faults appear to have little or no flow. Because regions with no flow are subjected to the same tectonic stresses as regions with flow, pore pressures must increase in the absence of fluid flow. Much of the fluid flowing in the shallow sediments appears to be forced through the sediment during porosity reduction with little, or no, additional fluid from external sources. The low- $\mathrm{Cl}$ concentration of some fluid at greater depths results from mixing between meteoric water, water released from clay and mineral dehydration and the original pore fluids. Methane concentrations suggest that only minor water would be released from gas-hydrate dissociation, and the $\mathrm{Br} / \mathrm{Cl}$ ratio indicates that membrane filtration is unimportant.

The New Hebrides sediment is composed of fractions of hydrous minerals larger than those of most other studied margins. Based on the $\mathrm{Cl}$ dilution, this source will provide only $\sim 0.05 \mathrm{~m}^{3} \mathrm{H}_{2} \mathrm{O} / \mathrm{m}^{3}$ sediments and even less if some of the dilution results from meteoric water mixing. Results from deep submersible dives (Greene et al., 1992) indicate that surface manifestations of fluid venting (e.g., sulfide-oxidizing benthic biota, carbonate crusts, and mud volcanoes) are sparse or missing completely from the margin, implying that there is a smaller fluid flux than from other previously studied margins. This apparently smaller flux could be caused by minimal recharge of meteoric water because of the short time of exposure of land in the region and small geographic exposure of the land. Other water sources, such as gas hydrate dissociation, also appear to be missing from the margin.

\section{ACKNOWLEDGMENTS}

I am grateful for the superb assistance provided by the crew and technical staff aboard the JOIDES Resolution, especially the two Leg 134 chemistry technicians, MaryAnn Cusimano and Mark Simpson, who not only helped with the analyses, but also made the cruise more pleasant. I thank the co-chief scientists, Jean-Yves Collot and Gary Greene for allowing me to take many extra, and occasionally extraordinarily long, samples that were extremely valuable for this paper. I also thank Gunter Lugmair and Doug Macdougall for the use of their laboratory for the $\mathrm{Sr}$ isotope measurements and Ellen Martin for her patience in teaching me the $\mathrm{Sr}$ isotope techniques, and for her support in general. The paper has been improved by numerous thoughtful suggestions by Steve Chambers, Joris Gieskes, and Miriam Kastner and careful and conscientious reviews by Gerard Blanc, Jean-Yves Collot, and an anonymous reviewer. This work was supported by a JOI-United States Science Advisory Committee (USSAC) grant.

\section{REFERENCES}

Bekins, B.A., and Dreiss, S.J., 1992. A simplified analysis of parameters controlling dewatering in accretionary prisms. Earth Planet Sci. Lett., 109:275-287.

Blanc, G., Boulegue, J., and Gieskes, J.R., 1991. Chemical evidence for advection of interstitial fluid in the sedimentary series of the Barbados accretionary complex (Leg 110). Oceanol. Acta, 14:33-49.

Blanc, G., Gieskes, J.M., Vrolijk, P.J., et al., 1988. Advection de fluides interstitiels dans les séries sédimentaires du complexe d'accrétion de la Barbade (Leg 110 ODP). Bull. Soc. Geol. Fr., 8:453-460.

Blanc, G., Hawkins, J., Parson, L.M., Allan, J., et al., 1991. ODP Leg 135 Pacifique: Bassin de Lau. Geochronique, 40:9.

Bray, C.J., and Karig, D.E., 1985. Porosity of sediments in accretionary prisms, and some implications for dewatering processes. J. Geophys. Res., 90:768-778.

Carson, B., Suess, E., and Strasser, J.C., 1990. Fluid flow and mass flux determinations at vent sites on the Cascadia margin accretionary prism. $J$. Geophys. Res., 95:8891-8898.

Chambers, S.R., and Cranston, R.E., 1991. Interstitial-water geochemistry of Kerguelen Plateau sediments. In Barron, J., Larsen, B., et al., Proc. ODP, Sci. Results, 119: College Station, TX (Ocean Drilling Program), 347374.

Collot, J.-Y., Daniel, J., and Burne, R.V., 1985. Recent tectonics associated with the subduction/collision of the d'Entrecasteaux zone in the central New Hebrides. Tectonophysics, 112:325-356.

Collot, J.-Y., and Fisher, M.A., 1991. The collision zone between the North d'Entrecasteaux Ridge and the New Hebrides Island Arc. Part 1: Seabeam morphology and shallow structure. J. Geophys. Res., 96:4457-4478.

Collot, J.-Y., Greene, H.G., Stokking, L.B., et al., 1991. Proc. ODP, Init. Repts., 134: College Station, TX (Ocean Drilling Program).

Colten-Bradley, V.A., 1987. Role of pressure in smectite dehydration-effects on geopressure and smectite-to-illite transformation. AAPG Bull., 71: 1414-1427.

COSOD II, 1987. Rep. 2nd Conf. Sci. Ocean Drilling, Strasbourg, France, (European Science Foundation).

Craig, H., 1961. Isotopic variations in meteoric waters. Science, 133:1702-1703.

Daniel, J., and Katz, H.R., 1981. d'Entrecasteaux zone, trench and western chain of the central New Hebrides Island Arc: their significance and tectonic relationship. Geo-Mar. Lett., 1:213-219.

\footnotetext{
Abbreviations for names of organizations and publications in ODP reference lists follow the style given in Chemical Abstracts Service Source Index (published by American Chemical Society).
} 
Dansgaard, W., 1964. Stable isotopes in precipitation. Tellus, 16:436-468.

Davidson, D.W., El-Defrawy, M.K., Fuglen, M.D., and Judge, A.S., 1978. Natural gas hydrates in northern Canada. Proc. 3rd Int. Conf. on Permafrost, 3:937-943.

Egeberg, P.K., and Leg 126 Shipboard Scientific Party, 1990. Unusual composition of pore-waters found in the Izu-Bonin fore-arc sedimentary basin. Nature, 344:215-218.

Elderfield, H., 1986. Strontium isotope stratigraphy. Palaeogeogr., Palaeoclimatol., Palaeoecol., 57:71-90.

Faure, G., 1986. Principles of Isotope Geology: New York (Wiley).

Fisher, M.A., Collot, J.-Y., and Geist, E.L., 1991. The collision zone between the North d'Entrecasteaux Ridge and the New Hebrides Island Arc. Part 2: structure from multichannel seismic data. J. Geophys. Res., 96:4479-4495.

Fowler, S.R., White, R.S., and Louden, K.E., 1985. Sediment dewatering in the Makran accretionary prism. Earth Planet. Sci. Lett., 75:427-438.

Gieskes, J.M., 1981. Deep-sea drilling interstitial water studies: implications for chemical alteration of the oceanic crust, layers I and II. In Warme, J.E., Douglas, R.G., and Winterer, E.L. (Eds.), The Deep Sea Drilling Project: A Decade of Progress. Spec. Publ.-Soc. Econ. Paleontol. Mineral., 32:149-167.

Gieskes, J.M., Gamo, T., and Brumsack, H.J., 1991. Chemical methods for interstitial water analysis aboard JOIDES Resolution. ODP Tech. Note, 15.

Gieskes, J.M., Gamo, T., and Kastner, M., 1993. Major and minor element geochemistry of interstitial waters of Site 808, Nankai trough: an overview. In Hill, I.A., Taira, A., Firth, J.V., et al., Proc. ODP, Sci. Results, 131: College Station, TX (Ocean Drilling Program).

Gieskes, J.M., Vrolijk, P., and Blanc, G., 1990. Hydrogeochemistry, ODPLeg 110: an overview. In Moore, J.C., Mascle, A., et al., Proc. ODP, Sci. Results, 110: College Station, TX (Ocean Drilling Program), 395-408.

Gill, J.B., 1981. Orogenic Andesites and Plate Tectonics: New York (Springer-Verlag).

Graf, D.L., 1982. Chemical osmosis, reverse chemical osmosis, and the origin of subsurface brines. Geochim. Cosmochim. Acta, 46:1431-1448.

Greene, H.G., Collot, J.-Y., Pelletier, B., and Lallemand, S., 1992. Observation of forearc seafloor deformation along the north D'Entrecasteaux RidgeNew Hebrides Island Arc collision zone from Nautile submersible. In Collot, J.-Y., Greene, H.G., Stokking, L.B., et al., Proc. ODP, Init. Repts., 134: College Station, TX (Ocean Drilling Program), 43-53.

Greene, H.G., and Wong, F.L. (Eds.), 1988. Geology and Offshore Resources of Pacific Island Arcs-Vanuatu Region. Circum-Pac. Counc. Energy and Miner. Resour., Earth Sci. Ser., 8.

Han, M.W., and Suess, E., 1989. Subduction induced pore fluid venting and the formation of authigenic carbonates along the Cascadia continental margin: implications for the global Ca-cycle. Palaeogeogr., Palaeoclimatol., Palaeoecol., 71:97-118.

Hesse, R., and Harrison, W.E., 1981. Gas hydrates (clathrates) causing pore water freshening and oxygen isotope fractionation in deep-water sedimentary sections of terrigenous continental margins. Earth Planet. Sci. Lett., 55:453-462.

Hodell, D.A., Mueller, P.A., and Garrido, J.R., 1991. Variations in the strontium isotopic composition of seawater during the Neogene. Geology, 19:24-27.

Holser, W.T., 1966. Bromide geochemistry of salt rocks. In Rau, J.L. (Ed.), Proc. 2nd Symp. on Salt. Ohio Geol. Soc., 248-275.

- 1970. Bromide geochemistry of some non-marine salt deposits in the southern Great Basin. Mineral. Soc. Am., Spec. Pap., 3:307-319.

1979. Trace element and isotopes in evaporites. Mineral. Soc. Am. Rev., 6:295-346.

Holser, W.T., and Wilgus, C.W., 1981. Bromide profiles of the Röt salt, Triassic of northern Europe as evidence of its marine origin. Neues Jahrb. Mineral. Mb., 6:267-276.

Kastner, M., Elderfield, H., and Martin, J.B., 1991. Fluids in convergent margins: what do we know about their composition, origin, role in diagenesis and importance for oceanic chemical fluxes? Philos. Trans. R. Soc. London A, 325:243-259.

Kastner, M., Elderfield, H., Martin, J.B., Suess, E., Kvenvolden, K.A., and Garrison, R.E., 1990. Diagenesis and interstitial-water chemistry at the Peruvian continental margin-major constituents and strontium isotopes. In Suess, E., von Huene, R., et al., Proc. ODP, Sci. Results, 112: College Station, TX (Ocean Drilling Program), 413-440.

Kastner, M., Martin, J.B., and Elderfield, H., 1989. The source of low chloride fluids in the upper slope of the Peruvian continental margin. In Epstein's 70th Birthday Symp. California Inst. Technol., 1-2.
Kharaka, Y.K., and Berry, F.A., 1973. Simultaneous flow of water and solute through geological membranes. I: experimental investigations. Geochim. Cosmochim. Acta, 37:2577-2603.

Lancelot, Y., Larson, R., et al., 1990. Proc. ODP, Init. Repts., 129: College Station, TX (Ocean Drilling Program).

Lawrence, J.R., and Gieskes, J.M., 1981. Constraints on water transport and alteration in the oceanic crust from the isotopic composition of pore water. J. Geophys. Res., 86:7924-7934.

Lawrence, J.R., Gieskes, J.M., and Broecker, W.S., 1975. Oxygen isotope and cation composition of DSDP pore water and the alteration of layer II basalts. Earth Planet. Sci. Lett., 27:1-10.

Le Pichon, X., Foucher, J.-P., Boulegue, J., Henry, P., Lallemant, S., Benedetti, M., Avedik, F., and Mariotti, A., 1990. Mud volcano field seaward of the Barbados accretionary complex: a submersible survey. J. Geophys. Res., 95:8931-8943.

LePichon, X., Henry, P., and the Kaiko Scientific Crew, 1991. Water budgets in accretionary wedges: a comparison. Philos. Trans. R. Soc. London A, 335:315-330.

Marthelot, J.-M., Chatelain, J.L., Isacks, B.L., Cardwell, R.K., and Coudert, E., 1985. Seismicity and attenuation in the central Vanuatu (New Hebrides) islands: a new interpretation of the effect of subduction of the d'Entrecasteaux fracture zone. J. Geophys. Res., 90:8641-8650.

Martin, J.B., Kastner, M., and Elderfield, H., 1991. Lithium: sources in pore fluids of Peru slope sediments and implications for oceanic fluxes. Mar. Geol., 102:281-292.

McDuff, R.E., and Gieskes, J.M., 1976. Calcium and magnesium profiles in DSDP interstitial waters: diffusion or reaction? Earth Planet. Sci. Lett., 33:1-10.

Moore, J.C., Mascle, A., Taylor, E., Alvarez, F., Andreieff, P., Barnes, R., Beck, C., Behrmann, J., Blanc, G., Brown, K., Clark, M., Dolan, J., Fisher, A., Gieskes, J., Hounslow, M., McClellan, P., Moran, K., Ogawa, Y., Sakai, T., Schoonmaker, J., Vrolijk, P.J., Wilkens, R., and Williams, C., 1987. Expulsion of fluids from depth along a subduction-zone decollement horizon. Nature, 326:785-788.

Mottl, M.J., 1992. Pore waters from serpentinite seamounts in the Mariana and Izu-Bonin forearcs, Leg 125: evidence for volatiles from the subducting slab. In Fryer, P., Pearce, J.A., Stokking, L.B., et al., Proc. ODP, Sci. Results, 125: College Station, TX (Ocean Drilling Program), 373-385.

Newman, A.C.D. (Ed.), 1987. Chemistry of Clays and Clay Minerals: New York (Wiley), Mineral. Soc. London Monogr., 6.

Parson, L., Hawkins, J., Allan, J., et al., 1992. Proc. ODP, Init. Repts., 135: College Station, TX (Ocean Drilling Program).

Robie, R.A., Hemingway, B.S., and Fisher, J.R., 1979. Thermodynamic Properties of Minerals and Related Substances at $298.15 \mathrm{~K}$ and 1 bar $\left(10^{5}\right.$ Pascals) Pressure and at Higher Temperatures. U.S. Geol. Surv. Bull., 1452.

Sand, L.B., and Mumpton, F.A. (Eds.), 1978. Natural Zeolites: Occurrence, Properties, Use: New York (Pergamon Press).

Savin, S.M., 1967. Oxygen and hydrogen isotope ratios in sedimentary rocks and minerals [Ph.D. dissert.]. California Inst. of Technol.

Seyfried, W.E., Janecky, D.R., and Mottl, M.J., 1984. Alteration of oceanic crust: implications for geochemical cycles of lithium and boron. Geochim Cosmochim. Acta, 48:557-569.

Taylor, F.W., 1992. Quaternary vertical tectonics of the central New Hebrides Island Arc. In Collot, J.-Y., Greene, H.G., Stokking, L.B., et al., Proc. ODP, Init. Repts., 134: College Station, TX (Ocean Drilling Program), 33-42.

von Huene, R., and Lee, H.J., 1983. The possible significance of pore fluid pressures in subduction zones. In Watkins, J.S., and Drake, C.L. (Eds.), Studies in Continental Margin Geology. AAPG Mem., 34:781-789.

Vrolijk, P., Chambers, S.R., Gieskes, J.M., and O'Neil, J.R., 1990. Stable isotope ratios of interstitial fluids from the Northern Barbados Accretionary Prism, ODP Leg 110. In Moore, J.C., Mascle, A., et al., Proc. ODP, Sci. Results, 110: College Station, TX (Ocean Drilling Program), 189-205.

Vrolijk, P., Fisher, A., and Gieskes, J., 1991. Geochemical and geothermal evidence for fluid migration in the Barbados accretionary prism (ODP Leg 110). Geophys. Res. Lett., 18:947-950.

Date of initial receipt: 16 April 1992

Date of acceptance: 30 October 1992

Ms 134S-008 